\title{
On the Role of the Pedunculopontine Nucleus and Mesencephalic Reticular Formation in Locomotion in Nonhuman Primates
}

\author{
(ㄴ)Laurent Goetz, ${ }^{1,2}$ Brigitte Piallat, ${ }^{1,2}$ @Manik Bhattacharjee, ${ }^{1,2}$ Hervé Mathieu, ${ }^{1,2,4,5}$ @Olivier David, ${ }^{1,2}$ \\ and $\odot$ Stéphan Chabardès ${ }^{1,2,3}$ \\ ${ }^{1}$ Université Grenoble Alpes, F-38000 Grenoble, France, ${ }^{2}$ Inserm, U1216, Grenoble Institut des Neurosciences, F-38000 Grenoble, France, ${ }^{3}$ Department of \\ Neurosurgery, Grenoble Alpes Hospital, Grenoble 38043, Cedex 9, France, ${ }^{4}$ Unité Mixte de Service IRMaGe, Centre Hospitalier Universitaire Grenoble, \\ Grenoble 38043, Cedex 9, France, and ${ }^{5}$ Unité Mixte de Service 3552, Centre National de la Recherche Scientifique, Grenoble 38043, Cedex 9, France
}

The mesencephalic reticular formation (MRF) is formed by the pedunculopontine and cuneiform nuclei, two neuronal structures thought to be key elements in the supraspinal control of locomotion, muscle tone, waking, and REM sleep. The role of MRF has also been advocated in modulation of state of arousal leading to transition from wakefulness to sleep and it is further considered to be a main player in the pathophysiology of gait disorders seen in Parkinson's disease. However, the existence of a mesencephalic locomotor region and of an arousal center has not yet been demonstrated in primates. Here, we provide the first extensive electrophysiological mapping of the MRF using extracellular recordings at rest and during locomotion in a nonhuman primate (NHP) (Macaca fascicularis) model of bipedal locomotion. We found different neuronal populations that discharged according to a phasic or a tonic mode in response to locomotion, supporting the existence of a locomotor neuronal circuit within these MRF in behaving primates. Altogether, these data constitute the first electrophysiological characterization of a locomotor neuronal system present within the MRF in behaving NHPs under normal conditions, in accordance with several studies done in different experimental animal models.

Key words: locomotion; mesencephalic locomotor region; mesencephalic reticular formation; microelectrode recording; nonhuman primates; pedunculopontine nucleus

\section{Significance Statement}

We provide the first extensive electrophysiological mapping of the two major components of the mesencephalic reticular formation (MRF), namely the pedunculopontine and cuneiform nuclei. We exploited a nonhuman primate (NHP) model of bipedal locomotion with extracellular recordings in behaving NHPs at rest and during locomotion. Different MRF neuronal groups were found to respond to locomotion, with phasic or tonic patterns of response. These data constitute the first electrophysiological evidences of a locomotor neuronal system within the MRF in behaving NHPs.

\section{Introduction}

More than 50 years ago, a locomotor center was discovered in the mesencephalic reticular formation (MRF) (Shik et al., 1966).

Received July 1, 2015; revised Feb. 17, 2016; accepted Feb. 22, 2016.

Author contributions: L.G., B.P., and S.C. designed research; L.G. performed research; L.G., B.P., M.B., and H.M. contributed unpublished reagents/analytic tools; L.G., M.B., and 0.D. analyzed data; L.G., 0.D., and S.C. wrote the paper.

This work was supported by grants from Medtronic and by France Parkinson Association. Sponsors had no role in the study design, data collection, data analysis, or data interpretation. The neurophysiology facility and the Grenoble MRI facility IRMaGe were partly funded by the French program Investissement d'Avenir run by the Agence Nationale pour la Recherche (Grant Infrastructure d'avenir en Biologie Santé, ANR-11-INBS-0006). We thank Vincente Dicalogero for animal care; Olivier Montigon for treadmill development and technical assistance; Albert Fenoy for critical reading and helpful comments; Patrick Mouchet in Grenoble for insightful discussions; Yann Thibaudier for assistance during experiments; André Parent in Québec City, Canada for the critical reading and helpful anatomical
Since this fundamental finding, the functional realm of the MRF was enlarged to the control of muscular tone, sleep-wake mechanisms, arousal, and REM sleep (Garcia-Rill, 1991; Inglis and Winn, 1995; Kobayashi and Isa, 2002). The MRF is mainly composed of the pedunculopontine nucleus (PPN, or nucleus tegmentalis pedunculopontinus) and the cuneiform nucleus (CfN, or

comments; and Suzanne N. Haber and her team in the Department of Pharmacology and Physiology, University of Rochester Medical Center for the valuable tissue processing and immunohistochemistry.

Prof. Chabardès is consultant for Medtronic and has received financial support from Medtronic for preclinical research in the field of deep-brain stimulation. The remaining authors declare no competing financial interests.

Correspondence should be addressed to Professeur Stéphan Chabardès, Clinique de neurochirurgie, Pôle PALCROS, CHU Michallon, BP 217, 38043 GRENOBLE Cédex 09, France. E-mail: SChabardès@chu-grenoble.fr.

DOI:10.1523/JNEUROSCI.2514-15.2016

Copyright $\odot 2016$ the authors $\quad 0270-6474 / 16 / 364917-13 \$ 15.00 / 0$ 
nucleus cuneiformis), which contain heterogeneous cell populations intermingled with fiber tracts (Wang and Morales, 2009; Martinez-Gonzalez et al., 2012). The precise anatomical delineations of the PPN and CfN and their terminologies remain unclear, especially in human and nonhuman primates (NHPs) and highly controversial (Alam et al., 2011). The PPN is reciprocally connected with a wide range of functionally diverse brain structures, including the basal ganglia (BG) (Lavoie and Parent, 1994a; Martinez-Gonzalez et al., 2011). In primates, projections from the BG and motor cortical areas cover a large area in the MRF (Shink et al., 1997; Matsumura et al., 2000; Rolland et al., 2009, 2011).

The original functional description of the MRF was related to transitions in brain states (Moruzzi and Magoun, 1949) and it was later identified as the $\mathrm{CH} 5$ cholinergic group (Mesulam et al., 1984, 1989), which is suggested to form the cholinergic arm of the reticular activating system (RAS). In rodents, different PPN neurons that send ascending and/or descending projections to thalamic and pontine nuclei modulate their activity during transition between wakefulness to sleep (Mena-Segovia et al., 2008; Ros et al., 2010). Furthermore, neurons of the PPN and intralaminar thalamic nuclei are thought to be involved in the maintenance of the optimal state of arousal required for task performance (Garcia-Rill, 1991; Kinomura et al., 1996; Steriade, 1996; Kobayashi et al., 2002). It was generally accepted that PPN and CfN constitute the mesencephalic locomotor region (MLR) (Garcia-Rill and Skinner, 1987a, 1987b; Ryczko and Dubuc, 2013), although some authors now consider this area as a "rythmogenic" center rather than a locomotor one (Garcia-Rill, 2015). Locomotion would depend on tonic and phasic modulation of the ponto-medullary reticular formation under the MLR control via parallel glutamatergic and cholinergic pathways (Le Ray et al., 2011). The only electrophysiological study of the MLR in NHPs was undertaken in decerebrated preparations (Eidelberg et al., 1981). Recently, lesion of the PPN cholinergic neurons in NHPs were found to induce locomotor and postural deficits (Karachi et al., 2010).

Based on a presumed MLR in humans and data suggesting a role of the PPN in the pathophysiology of Parkinson's disease (PD) (Pahapill and Lozano, 2000), deep-brain stimulation (DBS) of the PPN has been proposed to treat gait disorders (Mazzone et al., 2005; Plaha and Gill, 2005). However, several studies have pointed out the lack of experimental data regarding the role of the PPN and surrounding structures in the control of locomotion, in gait disorders, and its relevance as a DBS target in pathological context (Galvan and Wichmann, 2008; Ferraye et al., 2010; Alam et al., 2011; Pereira et al., 2011; Grabli et al., 2012; Ryczko and Dubuc, 2013). Interestingly, a recent study using optogenetic stimulation of the MLR in the mouse provided convincing results underlying a concurrent regulation of locomotion and brain state (Lee et al., 2014). In the present study, based on the fact that locomotion in primates is unique and relies mainly on a supraspinal control characterized by these animals' ability to use natural bipedal locomotion, we exploited a bipedal model of locomotion in NHPs (Goetz et al., 2012) by means of microelectrode recordings (MERs) to identify and characterize a locomotor circuit in behaving animals.

\section{Materials and Methods}

\section{Animals}

Two macaques (Macaca fascicularis; CRP) were used in this study (Monkey K: male, 9 years old, $9 \mathrm{~kg}$; Monkey T: male, 3 years old, $5.5 \mathrm{~kg}$ ). All experiments were performed in accordance with the recommendations of the European Community Council Directives of 1986 (86/609/EEC) and the National Institutes of Health's Guide for the Care and Use of Laboratory Animals and were approved by the Ethics Committee of Région Rhône-Alpes. Animals were kept with other congeners, allowing social behavior, in standard conditions of temperature $\left(23 \pm 1{ }^{\circ} \mathrm{C}\right)$, humidity ( $65 \pm 4 \%)$, and light ( $12 \mathrm{~h}$ light/dark cycle). They had access ad libitum to food and water and were given fresh fruits and vegetables every day. All animals included in this study naturally adopted bipedal posture in their cage or during locomotion with a handling stick. Bipedal locomotion was obtained after positive reinforcement training and procedures developed in our previous study (Goetz et al., 2012). Importantly, during locomotion, the primates were always able to perform precise voluntary movements with their forelimbs such as delousing while the hindlimbs were performing stepping. This indicated that the bipedal locomotion behavior was under the control of an automatic process. However, caution should be observed when extrapolating data from treadmill experimental conditiond to overground locomotion (Goetz et al., 2012) because we cannot assume that motivational aspects necessary to trigger locomotion in the natural environment (Sinnamon, 1993; Jordan, 1998) were fully controlled here. Nevertheless, the primates' cooperation in the task was evidenced by the fact that, at any time during a locomotion session, the animal was free to avoid the treadmill or not. For a schematic description of the bipedal locomotion model, see Figure $3 E$.

\section{MRI protocol}

A combined in vivo and ex vivo MRI protocol was used to improve deep-brain structures targeting and localization. MRI acquisitions were performed in the Grenoble MRI facility IRMaGE.

In vivo MRI protocol (3T). MRI sequences were performed preoperatively and postoperatively under general anesthesia (ketamine $7 \mathrm{mg} / \mathrm{kg}$, i.m., and xylasine $0.6 \mathrm{mg} / \mathrm{kg}$, i.m.) using a Philips 3T Achieva MRI scanner. Fast spin echo multislices dual-echo images were acquired in axial, coronal, and sagittal planes with $1.5 \mathrm{~mm}$ slice thickness and $0.45 \mathrm{~mm}$ in-plane resolution to localize the area of interest and to control the position of the implantable recording chamber.

Ex vivo protocol (3T). High-resolution MR images of the head were obtained postmortem after removal of the recording chamber and head holder using a Philips 3T Achieva MRI scanner ( $800 \mu \mathrm{m}$ slice thickness, $200 \mu \mathrm{m}$ in-plane resolution).

Ex vivo protocol (7T). High-resolution MR images of the macaque brain were obtained postmortem from perfused brains using Bruker Biospin 7020 USR 7T MRI scanner. Multislices fast spin echo methods were used. Images were acquired in axial, coronal, and sagittal planes with 480 $\mu \mathrm{m}$ slice thickness and $60 \mu \mathrm{m}$ in-plane resolution to obtain efficient $3 \mathrm{D}$ reconstruction allowing precise visualization in every direction (see Fig. $1 C-F)$.

\section{Surgical procedures}

The primate was maintained under general anesthesia by alternative intramuscular injection of ketamine $(7 \mathrm{mg} / \mathrm{kg}, \mathrm{i} . \mathrm{m}$.$) and xylasine (0.6 \mathrm{mg} /$ $\mathrm{kg}$, i.m.). Saline solution $(\mathrm{NaCl} 0.9 \%)$ was infused intravenously all along the duration of the surgery. The head was fixed on a stereotaxic frame and the body was laid on an electric blanket. Local anesthesia of the scalp and muscle was performed before the surgical incision. Bi-orthogonal $\mathrm{x}$-ray ventriculography (see Fig. $1 A, B$ ) was performed by injection of $2 \mathrm{ml}$ of contrast agent (Iopamidol $300 \mathrm{mg}$ Iode/ml) to define the stereotaxic coordinate system (Percheron, 1997). MRI-compatible titanium footed head holder and recording chamber (Crist Instrument) were stereotactically fixed to the skull with titanium screws. The center of the recording chamber was positioned vertical to the PC plane on the right hemisphere and was tilted $\sim 10^{\circ}$ laterally in the coronal plane. Three epidural titanium screw electrodes were implanted in the frontal and parietal brain region. Analgesic/anti-inflammatory therapies (ketoprofen, $2 \mathrm{mg} / \mathrm{kg}$, i.m.) and antibiotics (amoxicillin/clavulinic acid, $10 \mathrm{mg} / \mathrm{kg}$. i.m.) were provided during the 1 week postoperative period.

Imaging and histological assessment of microelectrode trajectories The precise assessment of microelectrode trajectories and recording site were performed with methods commonly used in NHP electrophysio- 
logical studies of the basal ganglia or PPN area in our laboratory and by other groups using ventricular landmarks (Wichmann et al., 1994; Matsumura et al., 1997; Devergnas et al., 2012). To improve the localization of microelectrode trajectories and recording sites in the brainstem, we combined these standard methods with MRI and histological data obtained for each primate. Data calculation and visualization of the recording site coordinates were computed using a preoperative MRI coregistered to the recording chamber position in relation with the $\mathrm{III}^{\mathrm{rd}}$ and $\mathrm{IV}^{\text {th }}$ ventricle landmarks as defined on the preoperative and postoperative ventriculography and further confirmed on ex vivo MRI sequences, electrolytic lesions, and immunohistolologic methods. More precisely, at the end of the surgery, a final biorthogonal X-rays were performed with minitubes inserted inside the microdrive setup used for the further MER sessions to calculate the trajectories of the microelectrodes in relation to the anterior commissure (AC) and posterior commissure (PC) by merging $\mathrm{X}$-rays and ventriculography according to the primate brain atlas (Martin and Bowden, 1996). Before euthanasia, electrolytic lesions were performed under general anesthesia with a direct current at $1 \mathrm{~mA}$ during 20 s (DS8000; WPI). Then, a new biorthogonal ventriculography with the microdrive containing minitubes was performed. Finally, the primate was deeply anesthetized with a lethal intravenous dose of pentobarbital $(3 \mathrm{ml} / 100 \mathrm{mg}$ ) and perfused through the left cardiac ventricle with saline solution followed by $4 \%$ paraformaldehyde (PFA 4\%) fixative solution. The head of the animal was removed and stored at $4^{\circ} \mathrm{C}$ in a PFA $4 \%$ bath during $3 \mathrm{~d}$ to perform the ex vivo 3T MRI protocol. The brain was removed and stored in a PFA $4 \%$ bath for 3 more days till the final ex vivo 7T MRI protocol. At the end of the MRI protocol, the brain was stored in phosphate buffer solution and cryoprotected in increasing gradients of sucrose $(10,20$, and finally $30 \%)$. Adjacent coronal serial sections of 50 $\mu \mathrm{m}$ from both primates cut on a freezing microtome were processed for either a Nissl stain or immunocytochemistry for choline acetyltransferase (ChAT).

\section{Visualization on MRI}

Neuronal activities were plotted as colored dots on 7T MRI sequences according to their stereotactic coordinates in the sagittal and coronal (perpendicular to AC-PC line) planes. In the axial plane, the distribution densities of the phasic and tonic responder neurons were computed as follows: neurons from the two primates were pooled together according to their stereotactic coordinates and were considered as a cloud of points. These points were convolved with an isotropic Gaussian kernel $(0.6 \mathrm{~mm}$ $\mathrm{SD}$, maximum amplitude of 1) and plotted on the 7T primate MRI in a transverse plane of the brainstem parallel to the ponto-mesencephalic junction (PMJ).

\section{MER procedures and data acquisition}

MER were performed using a microdrive setup (FlexMT and Multi Drive; Alpha Omega Engineering). Beveled minitubes were lowered through the dura matter to avoid any damage of the microelectrode when piercing the dura mater. Two to three tungsten microelectrodes (impedance: $2-3 \mathrm{M} \Omega$; FHC) were lowered independently. The extracellular signal was amplified, sampled at $50 \mathrm{kHz}$, and analogically bandpass filtered $(300-6000 \mathrm{~Hz})$. The ECoG signal was amplified, sampled at $6 \mathrm{kHz}$, and analogically bandpass filtered (1-300 Hz). EMG recordings were obtained simultaneously with MER from surface electrodes adapted to primate's morphology (Covidien). Before electrode placement, the skin was prepared by shaving and cleaning with an ether solution. EMG signals were amplified, sampled at $6 \mathrm{kHz}$, and band-pass filtered $(1-100 \mathrm{~Hz})$. EMG recordings were obtained from right and left hindlimb quadriceps muscle group (rectus femoris).

The first cortical neuronal activity defined the null depth of the trajectory. The precise electrophysiological mapping of the MRF was started from the caudal part of the inferior colliculus (IC, or colliculus inferior) to the pontine nuclei (nuclei pontis) just below the superior cerebellar peduncle (SCP, or pedunculus cerebellaris superior). Baseline activity was recorded for $1 \mathrm{~min}$ in bibedal standing position while the primate was awake, at rest and without any movement.

\section{Video recording and treadmill onset/offset markers}

The treadmill was controlled by a home-made software based on Labview system (National Instruments) that allowed us to tune the treadmill velocity and to synchronize the treadmill onset/offset with a visual preparation cue, electrophysiological recordings, and video acquisition using transistor-transistor logic inputs to the acquisition station. During locomotion sessions, videos were acquired perpendicular and parallel to the treadmill axis at 50 frame/s.

\section{Locomotion recording protocol}

A locomotion session was composed by the following three periods: prelocomotion (Pre-Loc) for $1 \mathrm{~min}$, locomotion (Loc) for $15 \mathrm{~s}(20 \mathrm{~s}$ in Primate T), and postlocomotion (Post-Loc) for $1 \mathrm{~min}$. Pre-Loc corresponded to the baseline neuronal activity while the primate was at rest in a standing position (bipedal posture). Treadmill velocity was $0.4 \mathrm{~m} / \mathrm{s}$. The electrophysiological (MER, ECoG, and EMG) and video recordings started simultaneously with the locomotion protocol sequence. A visual signal (LED light) placed in front of the primate was automatically activated $1.5 \mathrm{~s}$ before the treadmill onset and offset.

\section{Passive movement protocol}

At the end of each locomotion session, we evaluated the neuronal response in relation to passive movements of the two hindlimbs. This test consisted of flexion, extension, and rotation of the joints (ankle, knee, hip, wrist, and elbow). Furthermore, nonpainful cutaneous touch tests and manual pressure tests on the calf, thigh, and dorsal muscle were also performed.

\section{Signal processing and statistical analysis}

Spike-sorting processing. Isolation of the single-unit activity from the raw signal was performed using a template-matching algorithm (Spike 2; CED Software) to evaluate spike discharge characteristics; that is, the firing rate (FR) and firing pattern using standard methodology used in MER primate BG studies (Wichmann et al., 1994). Semiautomatic spike isolation was systematically verified and corrected on the basis of the quality of the spike waveforms and by principal components analysis. Calculation of the neuronal refractory period on interspike interval (ISI) was systematically performed and served as an exclusion criterion when it was found to be $<2 \mathrm{~ms}$ (Fee et al., 1996). The resulting spike train representing the activity of a single-unit neuron had to contain a minimum of 500 events to be included in the study. In the text, the terms "neuronal activity" or "neuron" always refer to single-unit neuronal activity.

Baseline FR analysis. FR analysis was performed using homemade software developed under MATLAB Station version 7.9.1-R2009b (The MathWorks). The baseline period corresponded to the period preceding the locomotion session (Pre-Loc) while the primate was in a bipedal standing position without any hindlimb or forelimb movement and wide awake. For each neuron, in each recording condition (i.e., baseline or locomotion), we used the ISI data to calculate the mean, SD, and median of the FR. Raster plots were constructed under Spike 2 software. To evaluate whether pause in the neuron discharge was frequent, we calculated the pause index as proposed by Favre et al. (1999) as the number of ISIs $>50 \mathrm{~ms}$ divided by the number of ISIs $<50 \mathrm{~ms}$.

Firing pattern analysis. We determined the firing pattern characteristics by a combined approach using data based on the interval ISI and autocorrelogram analysis further completed with burst detection methods (Abeles, 1982; Wichmann et al., 1994; Baron et al., 2011; Piallat et al., 2011). Autocorrelation histograms of spike trains were calculated for $1 \mathrm{~s}$ ( 1 ms bin width) using MATLAB software. Peaks were considered significant if they were found to be above the confidence line set at mean \pm 2 SDs. We classified single-unit activities as regular, irregular, or burst as follows: regular activity was characterized by a tonic discharge with a narrow peaked interval interspike distribution and a significant peak on the autocorrelation function usually equal to the mean firing period (i.e., 1/FR; see Fig. 2B). Irregular activity was characterized by a wide interval interspike distribution and a flat autocorrelogram (see Fig. 2C). Burst activity showed a wide or bimodal interval interspike distribution and a significant single peak on the autocorrelation function (see Fig. $2 A$ ). In addition, two different burst detection methods were used to complet the 
detection: the "Poisson surprise" method of Legéndy and Salcman (1985). Applied to our data, using the Poisson surprise method, bursts were defined as segments including at least 3 spikes with an $S$ value of at least 3. The Poisson surprise method was applied for each period of locomotion session. Due to the fact that some neurons displayed some long periods of pause that affected the mean FR, thus affecting the Poisson surprise $S$ value, we complemented the burst detection with the interval method that calculates the percentage of spikes in burst. The method that we used was derived from the study of Baron et al. (2011). For each neuron, independently of period of the locomotion session, we selected the following final parameters: maximum initial interval signifying burst onset: the median ISI during Pre-Loc period; longest ISI allowed within a burst: median ISI/2 during Pre-Loc period; Minimum number of events in a burst: 3 . This allowed us to use a burst detection method based on the baseline median rather than the mean FR. Based on these two methods and the complementary ISI and autocorrelogram analysis, the definition of the burst pattern group was statistically significant (mean surprise value) in the two primates using the two methods (data not shown).

FR analysis during locomotion. We developed different statistical tests using MATLAB Station version 7.9.1-R2009b (The MathWorks) to classify the neuronal response to locomotion according to the modulation of the FR during locomotion and nonlocomotion periods (Pre-Loc and Post-Loc) and/or in relation to quadriceps muscle group EMG activity. The latter was band-pass filtered between 100 and $200 \mathrm{~Hz}$ and rectified. Modulation of muscle activity was then obtained by convoluting the absolute value of the band-pass-filtered EMG with a Hanning window of $1 \mathrm{~s}$ duration. The main frequency of EMG modulation (EMGf) was estimated as the main frequency of the fast Fourier transform spectrum (frequency resolution of $0.05 \mathrm{~Hz}$ ). The main frequency of spike train modulation during locomotion (FqLoc) was obtained similarly. Neurons for which EMGf-FqLoc was $<0.1 \mathrm{~Hz}$ and FqLoc was $>0.1 \mathrm{~Hz}$ were classified as phasic responder neurons.

The remaining neurons were classified according to the modulation of their mean FR between Pre-Loc (FRmPreLoc), Loc (FRmLoc), and PostLoc (FRmPostLoc) periods. Three ratios of FR were used to describe the pattern of response as follows:

$$
\left(\frac{\text { FRmLoc }}{\text { FRmPreLoc }}\right) ;\left(\frac{\text { FRmPostLoc }}{\text { FRmPreLoc }}\right) ;\left(\frac{\text { FRmLoc }}{\text { FRmPostLoc }}\right)
$$

The null distribution of each ratio was estimated from surrogate data $(n=4999)$. Surrogate FR were obtained by randomly distributing the same number of spikes for each session and neuron assuming the null hypothesis of no temporal correlation between locomotion periods. Using a statistical threshold of $p<0.05$ from surrogate distribution, classes of neurons were then defined as follows:

Tonic increase responder neuron: a significant increase in FR during Loc versus Pre-Loc and no significant difference between Pre-Loc and Post-Loc FR.

Tonic decrease responder neuron: a significant decrease in FR during Loc versus Pre-Loc and no significant difference between Pre-Loc and Post-Loc FR.

Prolonged tonic increase responder neuron after Loc: a significant increase in FR during Loc versus Pre-Loc, no significant difference between Loc and Post-Loc, and a significant diffrence between Pre-Loc and Post-Loc.

Nonresponder: using the previous tests, no significant difference in FR was found between the three periods.

Other statistical analyses were performed using SPSS version 20 (IBM) and Prism (GraphPad Software) software. We decided to analyze the data obtained in the two primates separately assuming that the ability to adopt bipedal posture and locomotion and to develop automatic skills to walk bipedally on a treadmill after a training protocol was individual for each primate included in the protocol and could not reflect the overall M. fascicularis population. Therefore, we decided not to pool electrophysiological data obtained in the two primates, but rather to replicate the analysis and evaluate their similarities. However, we decided to pool data relative to the stereotaxic coordinates comparison obtained in the two primates because they are expressed in the same referential system.

Comparison of coordinates between groups of neurons was performed using Kruskal-Wallis one-way ANOVA. If the null hypothesis was rejected, post hoc tests were performed using the Mann-Whitney $U$ test with Bonferroni correction when number of comparisons was $<5$ (corrected threshold $=0.05 /$ number of comparisons) and Dunn's multiple-comparison test when the number of comparisons was $\geq 5$ to identify significant differences between groups. Comparison between two groups of coordinates were performed with the Mann-Whitney $U$ test. Statistical values are indicated in the text.

\section{Results}

\section{Electrophysiological mapping of the MRF at rest}

A total of 69 trajectories through the rostral brainstem were performed in two primates at rest (Primate $\mathrm{K}, n=30$; Primate T, $n=$ 39). The electrophysiological mapping extended anteroposteriorly from +2 to $-6 \mathrm{~mm}$ from the anterior border of PC. We explored an area between 1 and $7 \mathrm{~mm}$ laterally from the midline and rostrocaudally from the caudal level of the IC to the pontine nuclei below the SCP. To map the entire MRF, on the axial plan, we performed 1 trajectory every $1.4 \mathrm{~mm}^{2}$ and assessed the precise microelectrode position by calculating the stereotactic coordinates of each recording site using ventricular, MRI, and histological data (Fig. 1C,D).

For each trajectory, specific anatomical landmarks were identified by their electrophysiological signature and were reported on preoperative MRI to confirm the position of the microelectrode in the MRF. Our observations were similar to those from other electrophysiological recording studies of the same mesencephalic area (Matsumura et al., 1997; Kobayashi et al., 2002). The superior colliculus (colliculus superior) served as a marker for the entrance in the brainstem. The extent of the IC was easily recognizable and confirmed with auditory spike responses. Then, after a short period of silence, the entrance in the MRF was confirmed by the appearance of neuronal activities described previously in other electrophysiological studies in the same area in NHPs. The area of interest extended caudally to the fibers of the SCP easily identifiable as high-frequency positive spike bursts. Nevertheless, the presence of some neurons intermingled between those fibers could be detected. The transition from the mesencephalon to the pons was marked by a change in neuronal activity with typical "popcorn neurons" in the pons. The most medial trajectories were characterized by the recording of fibers or neuronal activities in relation to oculomotor movements due to activities of the nucleus of the trochlear nerve (nervus trochlearis) or fibers of the medial longitudinal fasciculus (fasciculus longitudinalis medialis). Laterally, fibers of the medial and lateral lemniscal system marked the lateral extent of the mesencephalon. The MRF was generally characterized by paucity and heterogeneity of neuronal activities. Typically, only a few neurons were recorded along a single microelectrode trajectory throughout the MRF. However, it was sometimes possible to record a cluster of neurons a few hundred micrometers apart. The neuronal density tended to increase as the microelectrode reached the caudal half of the area.

\section{Spike discharge characteristics: FR and pattern}

We recorded 235 single-unit activities in the MRF in baseline condition (91 neurons in Primate K and 144 neurons in Primate $\mathrm{T})$. Table 1 shows the spike discharge characteristics of the MRF neurons, and the distributions of the baseline mean FR are provided in Fig. $2 D$. In both animals, the majority of neurons had a 

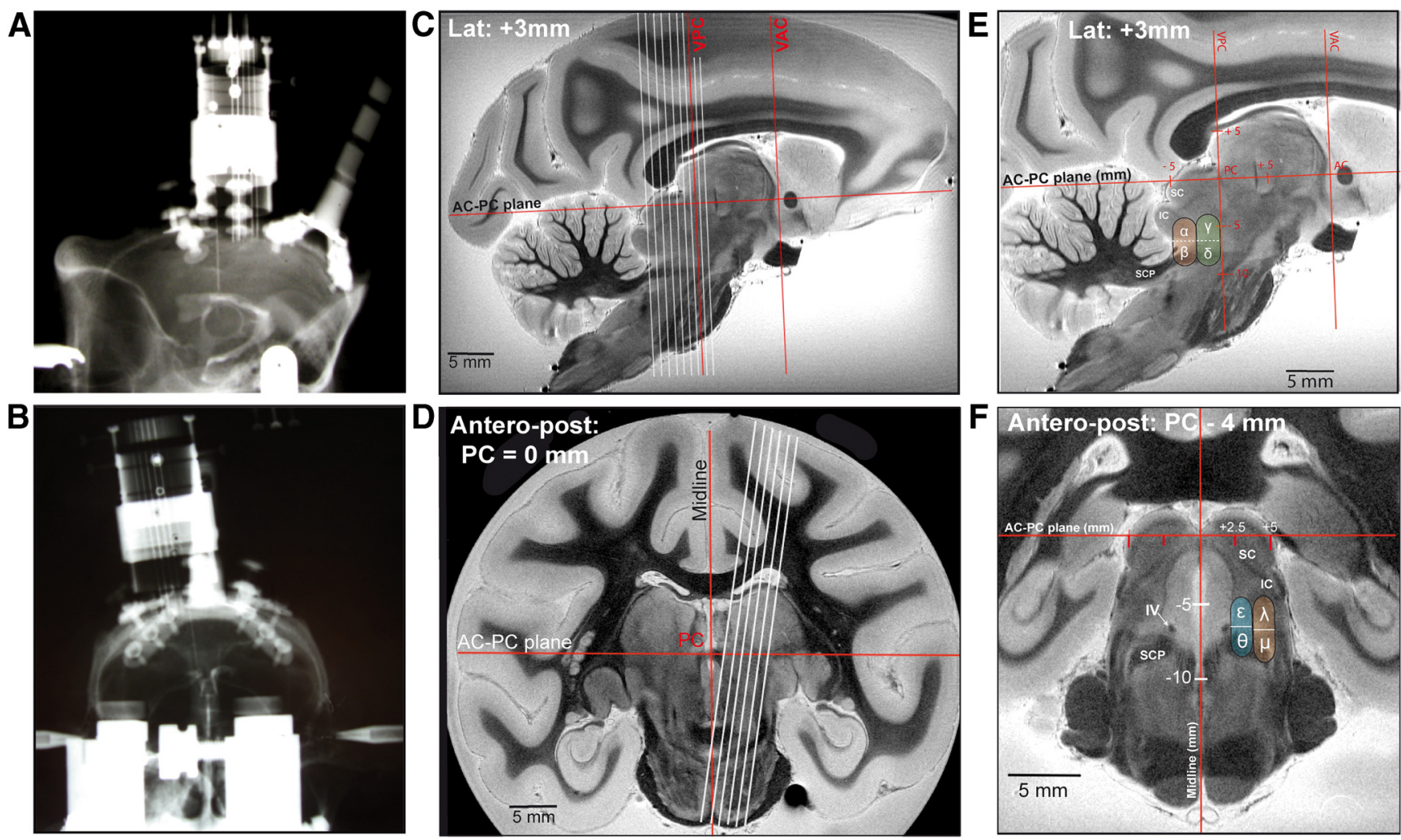

Figure 1. Multimodal targeting procedures. $\boldsymbol{A}, \boldsymbol{B}$, Biorthogonal ventriculography with the microdrive system. $\boldsymbol{C}, \boldsymbol{D}$, Reconstruction of the microelectrode trajectories (white diagonal lines) on postmortem $7 \mathrm{~T}$ MRl in sagittal and coronal planes according to III ${ }^{\text {rd }}$ ventricle landmarks (AC, $\left.P C\right)$. VAC, Vertical AC; VPC, vertical PC. $E$, $F$, Schematic representations of the different territories of the MRF on postmortem 7T MRI sagittal and coronal slices. Different parts of the MRF are as follows: $\alpha$, posterorostral; $\beta$, posterocaudal; $\gamma$, anterorostral; $\delta$, anterocaudal; $\varepsilon$, mediorostral; $\theta$, mediocaudal; $\lambda$, latero-rostral; $\mu$, latero-caudal. IV, Nucleus of the trochlear nerve.

Table 1. Baseline spike discharge characteristics in the two primates

\begin{tabular}{llllll}
\hline & & \multicolumn{3}{l}{ MRF } \\
\cline { 3 - 5 } & \multirow{2}{*}{$\begin{array}{l}\text { Number of } \\
\text { units }(n)\end{array}$} & & \multicolumn{3}{l}{ Baseline spike discharge activity } \\
\cline { 3 - 6 } & & Firing rate $(\mathrm{Hz})$ & Pause index & Pause ratio (ms) \\
\hline Primate K & 91 & Mean & 16.0 & 0.165 & 3.17 \\
& & SD & 15.7 & 0.13 & 3.17 \\
& & Median & 11.1 & 0.15 & 1.43 \\
Primate T & \multirow{2}{*}{144} & Range & {$[0.4 \sim 98.4]$} & {$[0 \sim 0.61]$} & {$[0 \sim 41.99]$} \\
& & Mean & 20.5 & 0.1 & 1.14 \\
& & SD & 17.7 & 0.11 & 2.46 \\
& & Median & 15.1 & 0.05 & 0.23 \\
& & Range & {$[0.5 \sim 90.1]$} & {$[0 \sim 0.53]$} & {$[0 \sim 20.03]$} \\
\hline
\end{tabular}

Baseline recording refers to the recording period while the primate was in bipedal standing position at rest, awake, and without any movement.

mean FR inferior to $40 \mathrm{~Hz}$, with variations extending from 1 up to $100 \mathrm{~Hz}$. Many neurons were characterized by pauses or silent periods in the spike discharge activity highlighted by the wide range of pause ratio and index in all conditions (Table 1), which ultimately influenced the mean FR. Figure $2, A-C$, provides examples of the three different patterns (irregular, regular, and burst) characterized by their firing properties (ISI and autocorrelogram). The relative proportion of neurons per pattern subgroup per primate is shown in Figure 2G. Interestingly, in addition to neurons firing irregularly, a large proportion of bursting neurons and neurons with a regular firing pattern were found in the two primates. In addition, by comparing the FR of the different pattern groups (Fig. $2 \mathrm{H}$ ), we found that regular neurons had a wide FR distribution and tended to have a higher FR than burst and irregular neurons.
Spatial distribution of the discharge FR and patterns

According to the $X, Y$, and $Z$ coordinates (AC-PC stereotactic system) of each recording site, in the two primates (pooled data), the distribution of the neuron coordinates in relation to the baseline FR band frequency of $10 \mathrm{~Hz}$ did not show any regional differences in baseline FR, except for a significant post hoc test difference in anteroposteriority for FR between 40 and $50 \mathrm{~Hz}$ compared with some other FR bands (Fig. 2E).

In regard to the firing pattern groups in relation to the coordinate distribution in the two primates (Fig. $2 F$ ), no significant difference in laterality was found between patterns $(p=0.3692)$, but there was a significant difference occurred in the $Y$ anteroposterior coordinates $(p<0.001)$. When looking at post hoc tests with Bonferroni-corrected threshold, we found that burst and irregular neurons were located more anterior than the regular neurons ( $p<0.0001$ and $p<0.005$, respectively). However, no significant difference was found between the $Y$ coordinates of the burst and irregular neurons $(p=0.018)$. A significant difference in the $Z$ rostrocaudality coordinates was noted between groups $(p=0.0289)$. Post hoc tests indicated that only burst neurons were significantly located more caudal than the regular neurons $(p=0.0162)$. No significant difference was found between $Z$ coordinates of the burst and irregular neurons $(p=0.441)$ or between irregular and regular neurons $(p=0.294)$.

Electrophysiological mapping of the MRF during locomotion In both primates, the MERs were started only when locomotion was under automatic control; that is, "nonvoluntary" (See Materials and Methods). We could record and analyze 255 single-unit activities during locomotion (91 in Primate K and 144 in Primate 


\section{A}

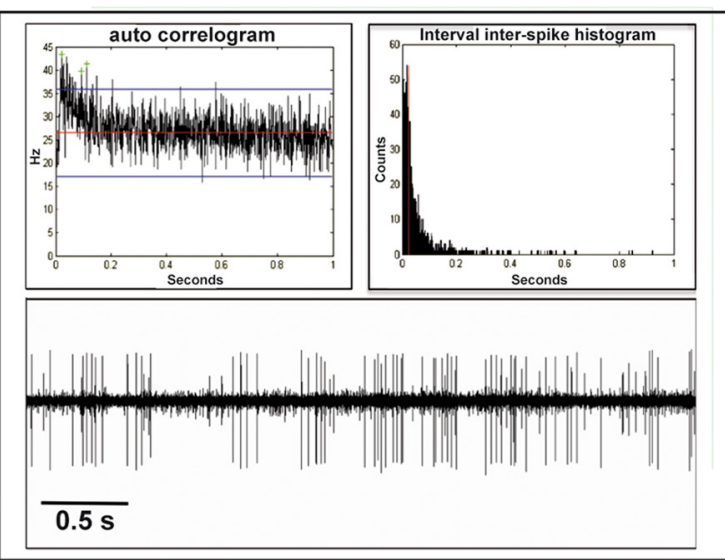

C

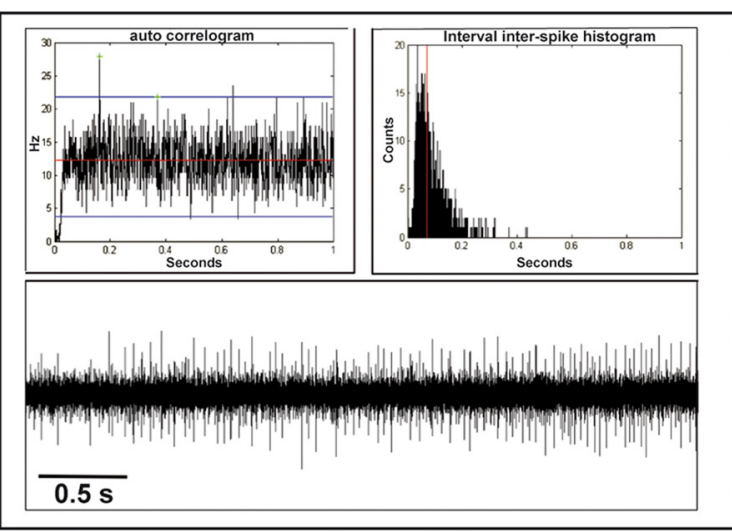

E

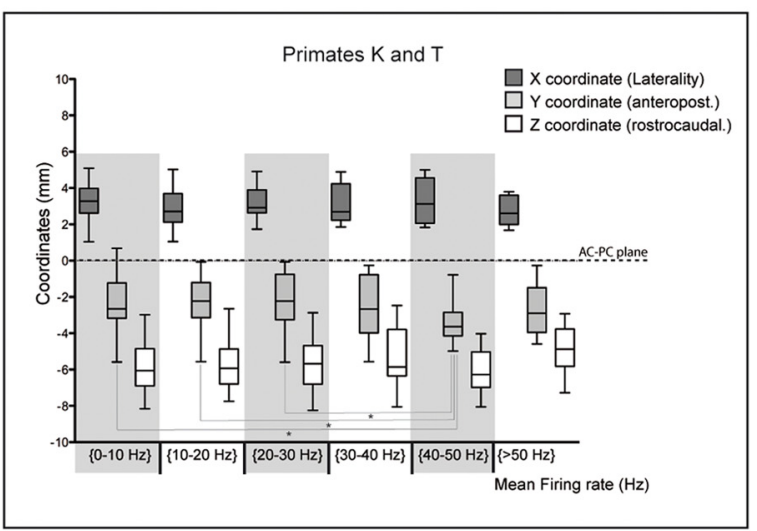

G

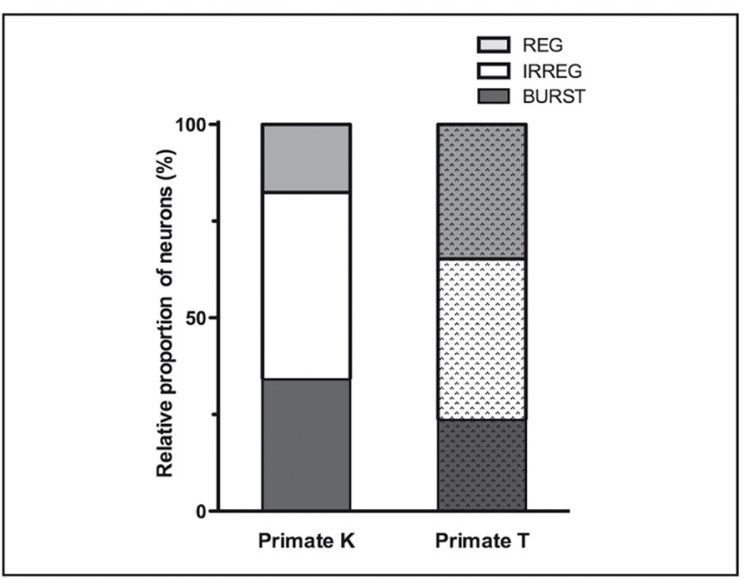

B

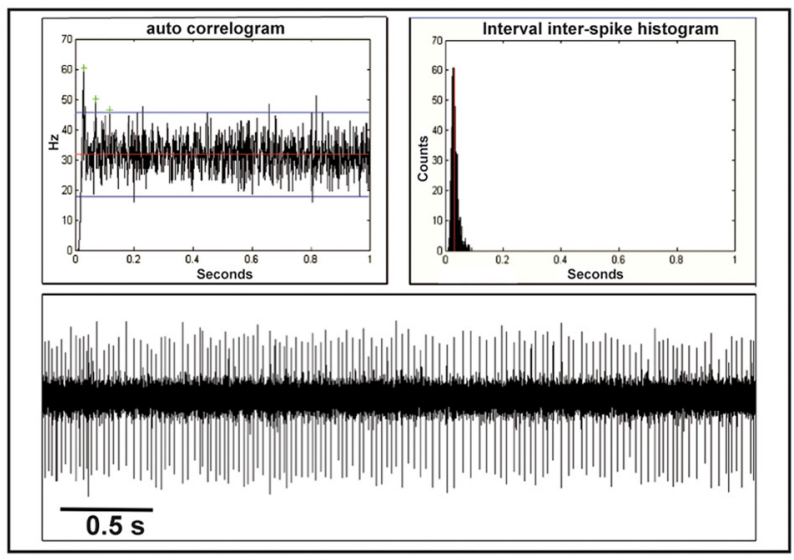

D
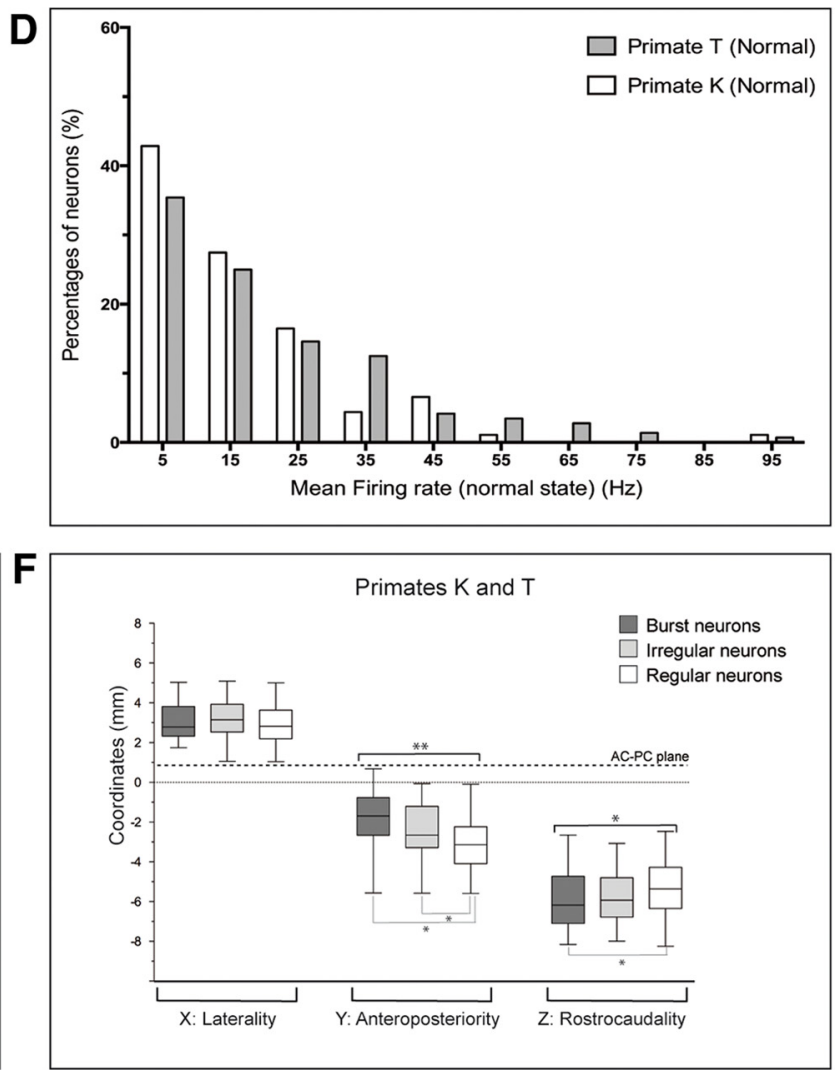

$\mathbf{H}$

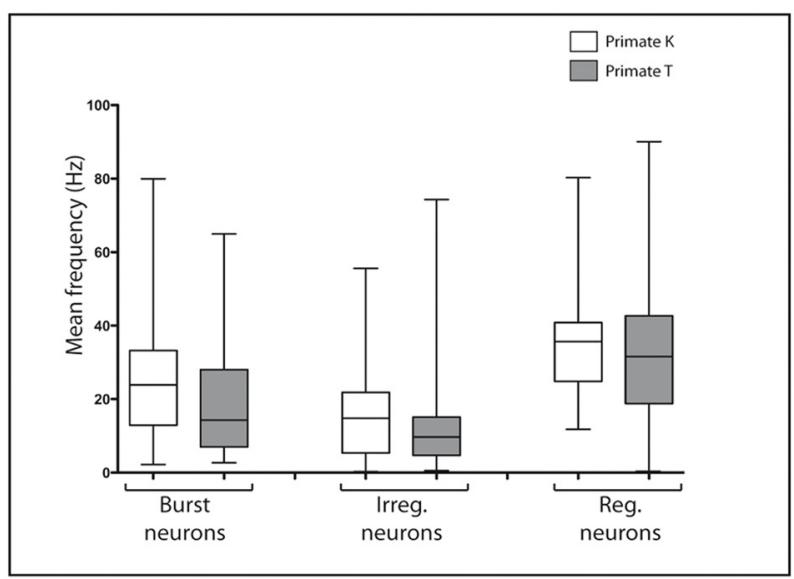

Figure 2. Baseline spike discharge characteristics. $\boldsymbol{A}-\boldsymbol{C}$, Neuronal pattern characteristics of the different subgroups. $\boldsymbol{A}$, Burst neuron characterized by a significant single peak in auto correlograms (ACr) and a wide ISI distribution. B, Regular neuron characterized by a narrow ISI distribution and a typical ACr with a first significant narrow peak followed by series of smaller peaks. $\boldsymbol{C}$, Irregular neuron characterized by a flat ACr and wide ISI distribution. Left upper corner of images, ACr; right upper corner, ISI. Bottom half are raw data. D, Distribution of (Figure legend continues.) 
T). During locomotion, the majority of the responding neurons had an FR $>15 \mathrm{~Hz}$ corresponding to the beta-/gamma-band activity. Statistical tests allowed us to define three different groups of cells: phasic, tonic, and nonresponder neurons. The evolution of the FR during locomotion is shown in Figure 3, $C$ and $D$, and the number and percentage of neurons per group of response during locomotion session is provided in Table 2.

Phasic responder neurons

A significant proportion $(36.3 \%, n=33$, and $17.4 \%, n=25)$ of the neurons recorded during locomotion in Primate $\mathrm{K}$ and Primate $\mathrm{T}$, respectively, changed their spontaneous spike discharge activity into a rhythmic activity characterized by discharge of bursts of spikes (Table 2). In those 58 neurons, the rhythmicity (i.e., the frequency of bursts) was time locked with the quadriceps EMG rhythmicity. Figure $3 A$ shows an example of the evolution of spike discharge activity of one phasic responder neuron. Raster plots of the phasic responders aligned on the treadmill onset are displayed in Figure 4, $A$ and $B$. Interestingly, some neurons were silent or had a very low activity before and after the locomotion, whereas other neurons had a sustained tonic activity at rest and shifted to a phasic rhythmic spike discharge activity during locomotion.

\section{Tonic responder neurons}

Based on statistical analysis, we could define three subgroups that represented 122 neurons: "tonic increase," "tonic decrease," and "tonic prolonged increase" responder neurons. The tonic increase responder neurons were defined by an increase of the FR during locomotion and by a decrease in frequency after the end of the locomotion session ( $p<0.05$, permutation test) and represented $29.7 \%$ $(n=27)$ and $33.3 \%(n=48)$ of the recorded neurons in Primate K and Primate T, respectively (Table 2). An example of a tonic increase neuron is provided in Figure 3B. Raster plots of the tonic increase responders aligned on the treadmill onset are provided in Figure 4, $C$ and $D$.

In Primate K and Primate T, $11 \%(n=10)$ and $14.6 \%(n=21)$ of the recorded neurons, respectively, were classified as tonic decrease responders due to their lower FR during the locomotion period compared with the Pre-Loc and Post-Loc periods ( $p<0.05$, permutation test). The tonic prolonged increase responder neurons $(4.4 \%$, $n=4$, in Primate $\mathrm{K}$ and $8.3 \%, n=10$, in Primate T) shared the characteristics of the tonic increase ones because they significantly increased their FR during locomotion ( $p<0.05$, permutation test). However, unlike the tonic increase responders, the tonic prolonged increase responders maintained a similar prolonged increased response at least during the $10 \mathrm{~s}$ Post-Loc period similar to that during the Loc period.

\section{Nonresponder neurons}

A total of 55 neurons $(18.6 \%, n=17$, in Primate $\mathrm{K}$ and $26.4 \% n=$ 38 , in Primate T) did not significantly modify their FR during locomotion and were considered "nonresponders" (Table 2).

$\leftarrow$

(Figure legend continued.) single-unit FR in the two primates. $y$-axis represents the number of neurons and $x$-axis represents the mean FR. The center of the first bin is at $5 \mathrm{~Hz}$ and the bin width is $10 \mathrm{~Hz}$. E, Distribution of the $X, Y$, and $Z$ coordinates (in millimeters) of the single-unit $F R$ per $10 \mathrm{~Hz}$ bin. *Significant $p<0.05$, post hoc test, Mann-Whitney $U$ test with Bonferroni corrected threshold. $F$, Distribution of the $X, Y$, and $Z$ coordinates (in millimeters) of the neuron pattern subgroups. ${ }^{*}$ Significant $p<0.01$, post hoc test; $p<0.01$, Mann-Whitney $U$ test with Bonferroni-corrected threshold. $G$, Relative proportion of neuron per pattern group in the two primates. $\boldsymbol{H}$, Mean FR per pattern group. In $\boldsymbol{E}-\boldsymbol{G}$, the box plots summarize the distribution of the neurons coordinates or $\mathrm{FR}$, with the limits of the box representing the 25 th and 75 th quartiles and the central line showing the median sample value. The whiskers extend to the minimum and maximum values. For baseline recordings, see Table 1 and Materials and Methods.
Passive movement response

All recorded neurons were tested for passive movement response. A total of 11 neurons clearly responded to passive flexion/extension of the leg or to manual pressure of calf or thigh muscle groups. In rare cases, the response was related to pressure on the lower dorsal muscle. Based on their electrophysiological characteristics, neurons were clearly differentiated from fibers of the medial lemniscus or the SCP. The response could be obtained by bilateral passive movement, but prevailed on the side ipsilateral to the recording site. Importantly, those passive movement neurons were all included in the phasic group.

\section{Anatomical location of neuronal responses to locomotion}

Knowing the exact $X, Y$, and $Z$ stereotactic coordinates of every recorded neuron, it was possible to determine and compare their precise positions within the MRF. Their location was displayed onto a 7T MRI brain template using the same stereotaxic anatomical landmarks and with the corresponding histological sections obtained from the two primate brains (Fig. 5). Instead of forming distinctive functional units, locomotion-responding neurons were scattered throughout the MRF. When considering the coordinates of the phasic responders versus the tonic responders neurons in the two primates (Fig. $3 F$ ), we found that the former tended to be located significantly more anterior and more rostral than the latter $(p=$ 0.0011 and $p=0.0016$, respectively). Table 3 provides more details on the spatial distribution of the responding neurons in the subareas of the MRF. It reveals that $57.2 \%$ of phasic responder neurons occurred throughout the rostro-caudal extent of the anteromedial part of the MRF, which corresponds to the PPN pars dissipata and the caudal aspect of the CfN, whereas the rest of this neuronal population was scattered in the MRF. Indeed, visualization on MRI shows that a large proportion of phasic neurons (red spheres) were distributed in the anterior part of the MRF just posterior to PC (Fig. 5A1$A 3, B 1-B 3)$. At PC $1.5 \mathrm{~mm}$, phasic neurons were more densely distributed along the border of the SCP. Positive passive lower limb movement neurons (yellow spheres), which also belong to the phasic group, were intermingled with the latter ones.

The tonic responder neurons were chiefly scattered in the posterior MRF (57.4\%; Table 3), a region that harbors the PPN pars dissipata and compacta and the posterior part of the CfN (Fig. 5C1$C 3, D 1-D 3)$. The use of ChAT immunostaining has allowed the identification of the cholinergic PPN neurons (ChAT+) (Fig. 5E2$E 3, F 2, F 3, G 2, G 3$ ) and their mapping on the corresponding MRI (Fig. $5 E 1, F 1, G 1)$. A higher density of cholinergic neurons was found in the posterior MRF corresponding to the PPN pars compacta (Fig. 5G2,G3).

Axial images parallel to the PMJ allowed us to visualize the region with the highest density of phasic and tonic neurons (Fig. $5 H 1, H 2, I 1-I 3, J 1, K 1, K 2)$. Interestingly, both groups of neurons were mainly located in the caudal MRF at the level of the PMJ. Although the neuronal populations partly overlapped, the density of tonic neurons was slightly higher in the posterior part of the MRF (encompassing the CfN and the PPN), whereas phasic neurons prevailed in the anterior part of the MRF (encompassing the PPN and the anterior CfN, which is referred to, in humans at this level, as the subcuneiform nucleus).

\section{Discussion}

The present study has provided the first electrophysiological mapping of the MRF in behaving primates at rest and during bipedal locomotion. Different neuronal populations were found to display phasic or tonic firing pattern during locomotion, thus confirming the existence of a locomotor neuronal circuit in the primate MRF. 

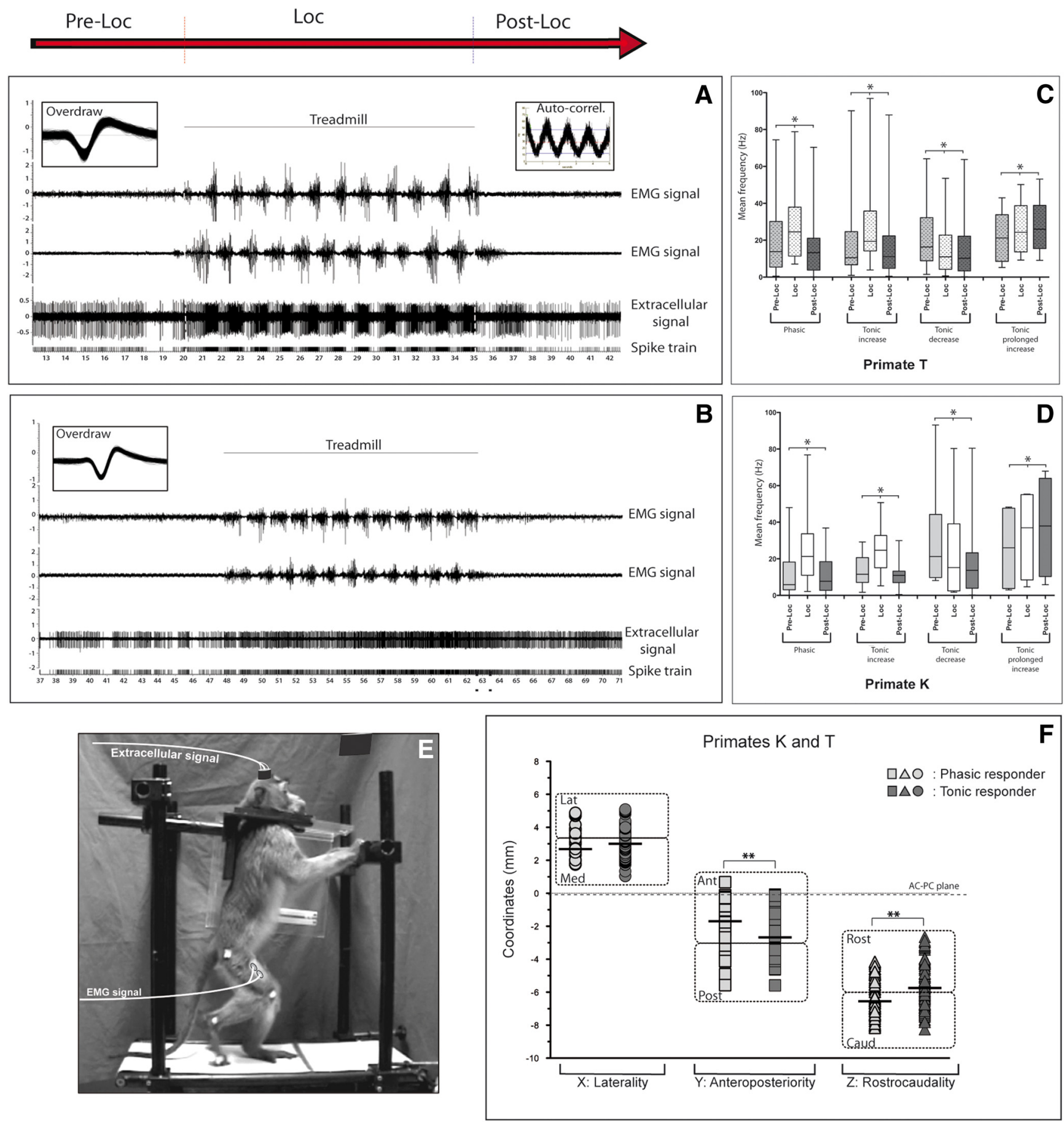

Figure 3. Neuronal response to locomotion. $\boldsymbol{A}, \boldsymbol{B}$, Examples of a phasic $(\boldsymbol{A})$ and a tonic increase $(\boldsymbol{B})$ responder neurons. The red arrow at the top of $\boldsymbol{A}$ indicates the events scale of a recording session. In $\boldsymbol{A}$ and $\boldsymbol{B}$, the first row displays the onset/offset treadmill information (transistor-transistor logic, or TTL, input). The second and third rows represent the raw EMG activities of the left and right quadriceps muscle group, respectively. The fourth and fifth rows represent the raw neuronal activity and the neuronal spike train after single-unit isolation, respectively. The window in the left upper corner shows the spike waveform of the neuron. The window in the right upper corner in $A$ shows the auto-correlogram of the neuron with typical oscillations in relation with the phasic feature of the spiking discharge during locomotion. $C, D$, Evolution of the FR during the three periods of a locomotion session per group. ${ }^{*} p<0.05$. The box plots summarize the distribution of discharge frequency (see Fig. $2 E, G$ ). $\boldsymbol{E}$, Schematic representation of the recording procedure during bipedal locomotion. $\boldsymbol{F}$, Diagram representation of the $X, Y$, and $Z$ coordinates of every responding neuron during locomotion. Light and dark symbols represent the phasic and tonic responders, respectively, during locomotion. On each diagram, the black line represents the median (**significant $p<$ 0.01, Kruskal-Wallis test). Lat, Lateral part of the MRF; Med, medial part of the MRF; Ant, anterior part of the MRF; Post, posterior part of the MRF; Rost, rostral part of the MRF; Caud, caudal part of the MRF as represented in Figure $1, E$ and $F$.

Electrophysiological characteristics of the MRF

We did not observe a clear electrophysiological distinction between the PPN and CfN, both structures being composed of sparse and rather physiologically heterogeneous neuronal populations, as already reported for human and NHPs (Mesulam et al.,
1984, 1989; Manaye et al., 1999). The single-unit activities were characterized by a wide FR distributions, similar to that reported in primates (Matsumura et al., 1997; Kobayashi et al., 2002; Okada and Kobayashi, 2015) and in PD patients (Weinberger et al., 2008; Piallat et al., 2009; Shimamoto et al., 2010) without any 
Table 2. Number of neurons (single unit) and relative proportion of the different groups of spike response to locomotion

\begin{tabular}{|c|c|c|c|c|c|c|}
\hline & & Locomotion & rge response, $n$ & & & \\
\hline & Number of units $(n)$ & Phasic & Tonic increase & Tonic decrease & Tonic prolonged increase & No response \\
\hline Primate K & 91 & $33(36.3 \%)$ & $27(29.7 \%)$ & $10(11 \%)$ & $4(4.4 \%)$ & $17(18.6 \%)$ \\
\hline Primate T & 144 & $25(17.4 \%)$ & $48(33.3 \%)$ & $21(14.6 \%)$ & $12(8.3 \%)$ & $38(26.4 \%)$ \\
\hline
\end{tabular}

Phasic responder neurons
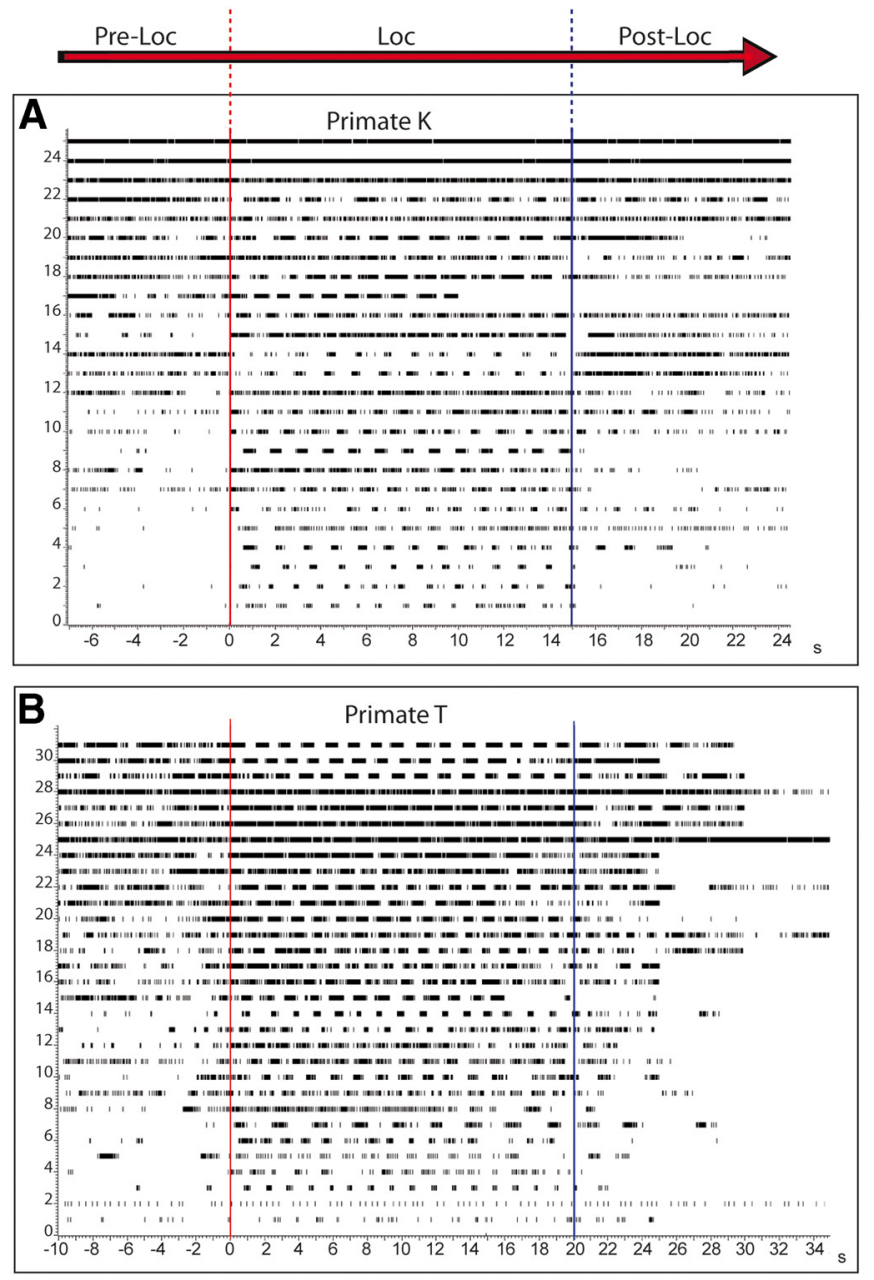

Tonic increase responder neurons
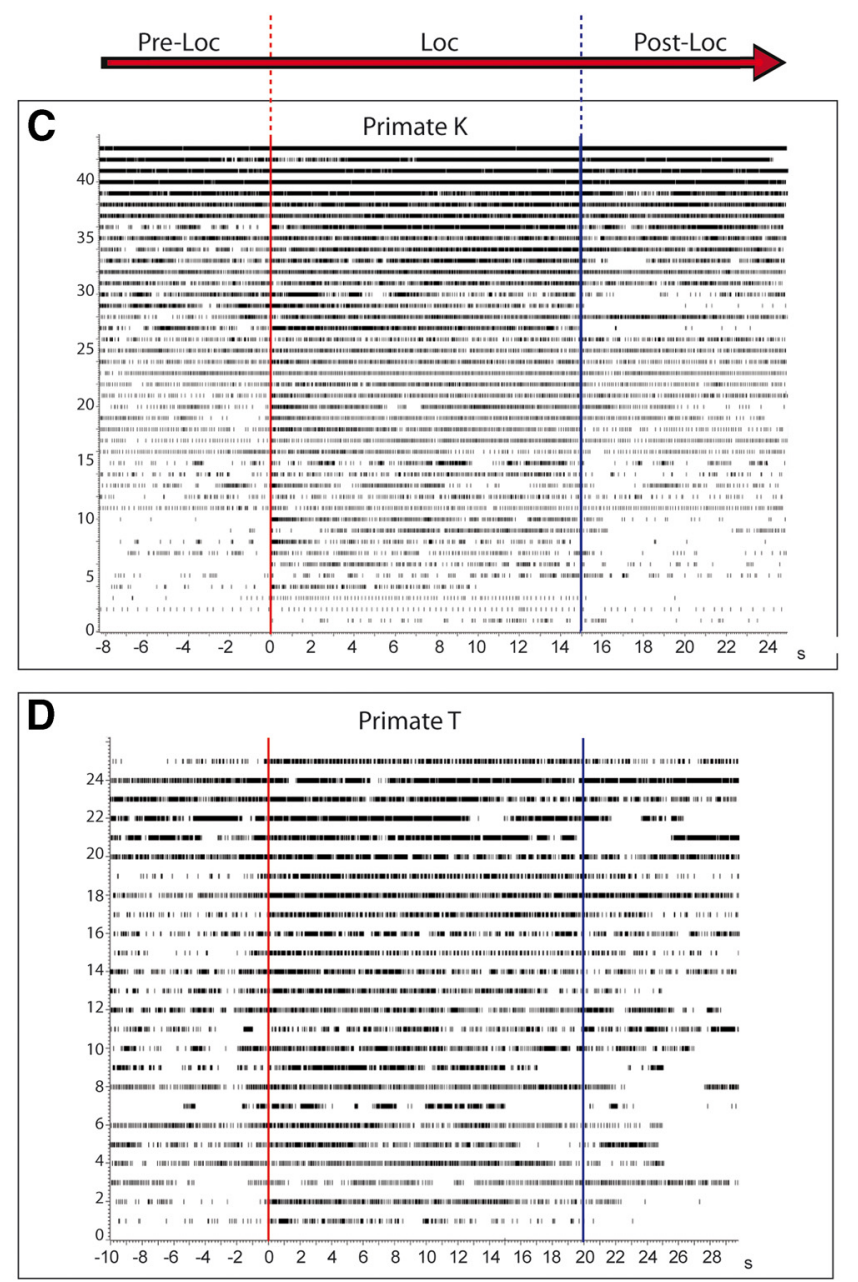

Figure 4. Raster plot during locomotion. The four panels represent the raster plot of the phasic $(\boldsymbol{A}, \boldsymbol{B})$ and of the tonic increase responder $(\boldsymbol{C}, \boldsymbol{D})$ neurons in the two primates. Each row represents the spike train of a neuron during a locomotion session. Spike trains are aligned on the treadmill onset (vertical red line). The $x$-axis represents time in seconds.

spatial correlation. Although the different neuronal populations overlapped, a higher proportion of burst neurons was found in the anterior MRF, whereas regular neurons, which could correspond to the putative cholinergic PPN type II neurons in rat due to their firing pattern similarities (Kang and Kitai, 1990; Takakusaki and Kitai, 1997), were often found intermingled with other nonregular neurons in the posterior aspect of the MRF and could be part of the so-called PPN pars compacta.

\section{Functional implication for locomotion}

In primate, clues for MLR are limited to decerebrate preparations (Eidelberg et al., 1981) and lesioning studies (Karachi et al., 2010). Here, in the behaving primate, we provide electrophysiological evidence for the existence of a locomotor circuit distributed within the MRF. Indeed, neurons with rhythmic discharge patterns coherent with EMG oscillations have been regarded as a way to control step frequency (Garcia-Rill and Skinner, 1988), strengthening the hypothesis of a rhythmic activation of pontomedullary tracts containing reticulo-spinal (RS) neurons that ultimately drives the cord to control the cadence of stepping (Drew et al., 1986). Some neurons were found to modulate their activity tonically during locomotion similar to the so-called "on/off" PPN neurons recorded in decerebrated preparations (Garcia-Rill and Skinner, 1988) and confirming data in PD patients during DBS implantation (Piallat et al., 2009). Those neurons could be involved in the duration of stepping or muscular tone to initiate or terminate locomotion via activation of the spinal circuitry by the RS system (Armstrong, 1988). Neurons with a prolonged increase response could represent a subpopulation involved in a complementary function, such as the retention of attention after locomotion. This response was not reported in decerebrate preparation studies, possibly as a consequence of the precollicu- 

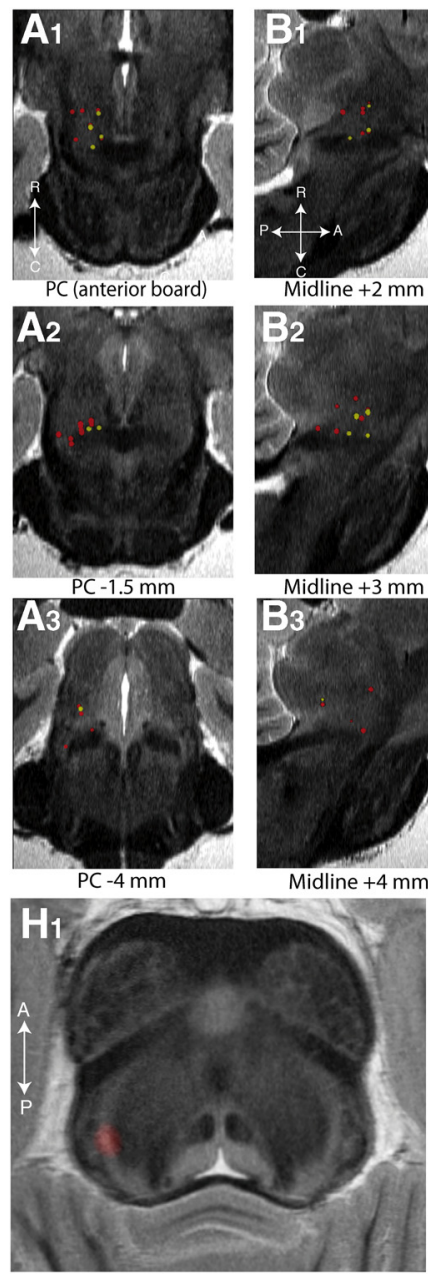

Phasic - PMJ -1 mm

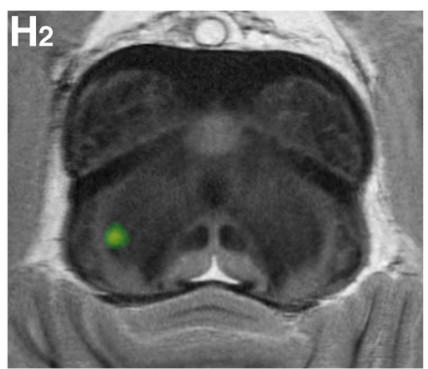

Tonic increase - PMJ $1 \mathrm{~mm}$

Midline $+4 \mathrm{~mm}$
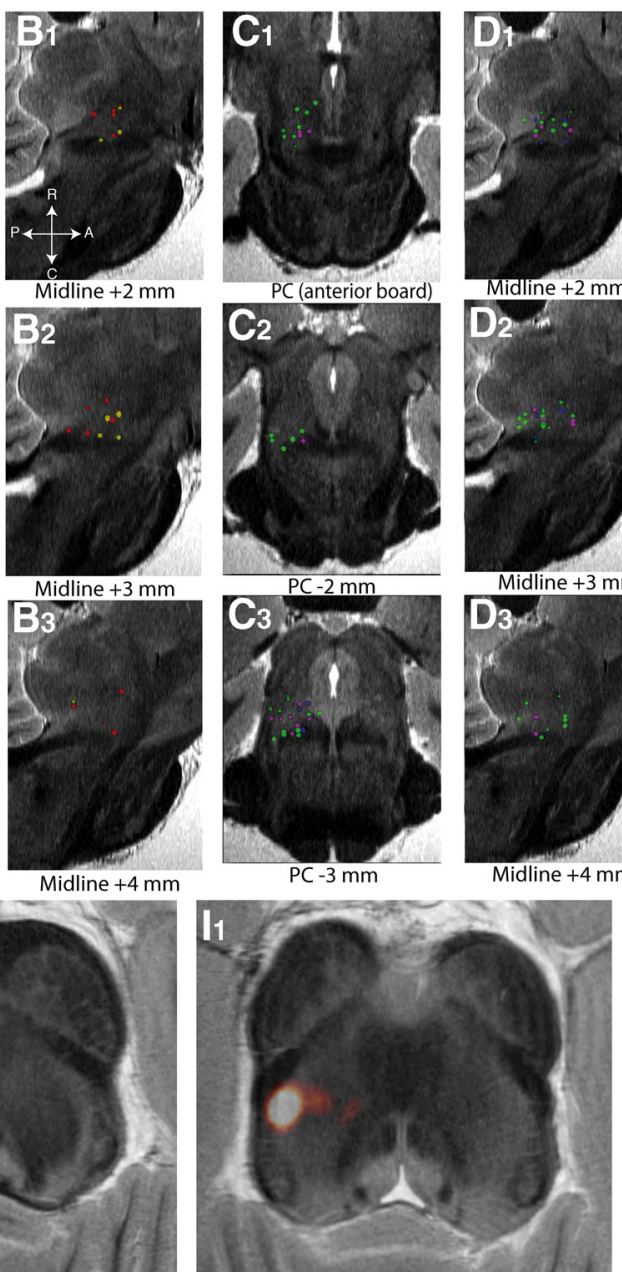

Phasic - PMJ

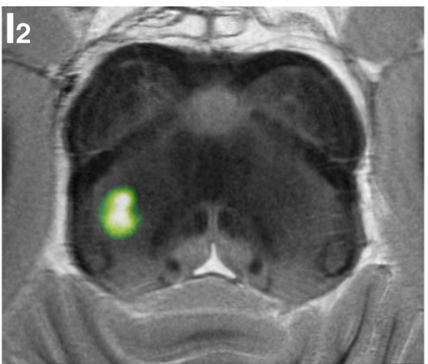

Tonic increase - PMJ
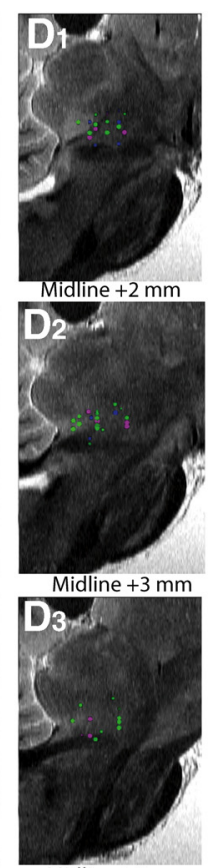

Midline $+4 \mathrm{~mm}$
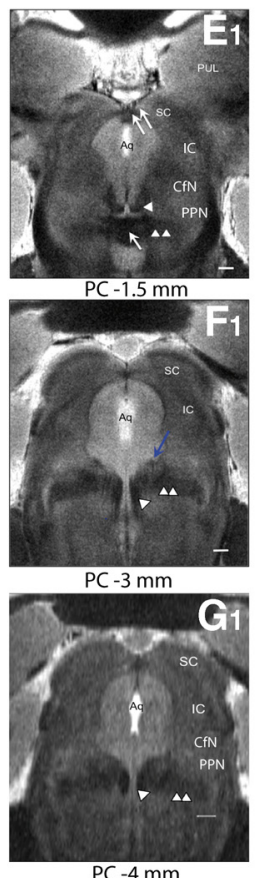

$\mathrm{PC}-4 \mathrm{~mm}$

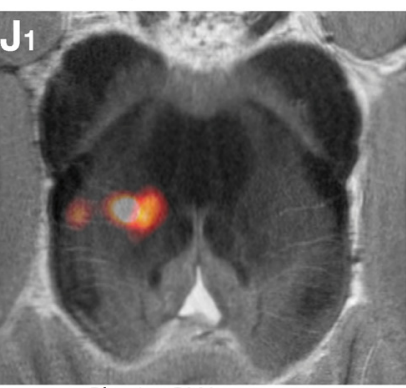

Phasic - PMJ +0.5 mm

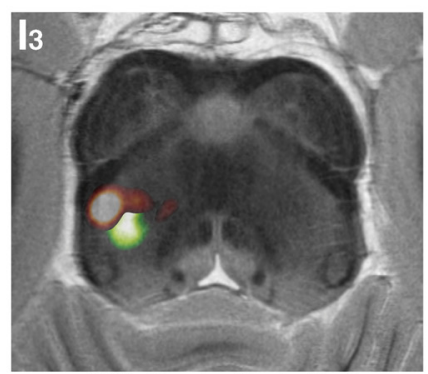

PMJ - Overlay Phasic /Tonic
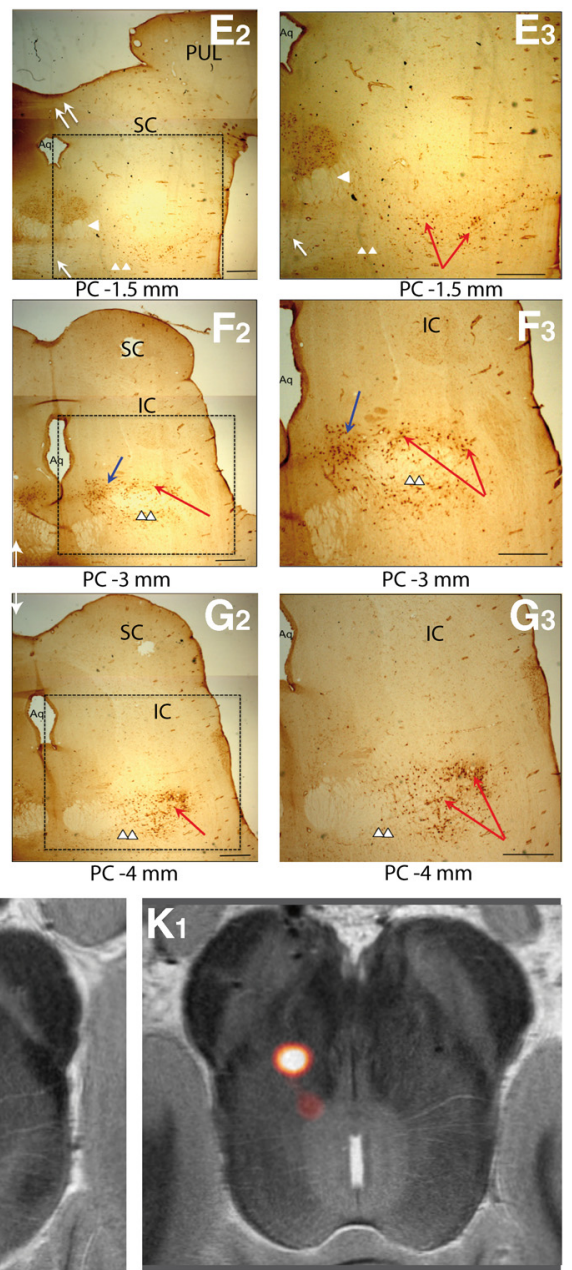

Phasic - PMJ +2 mm

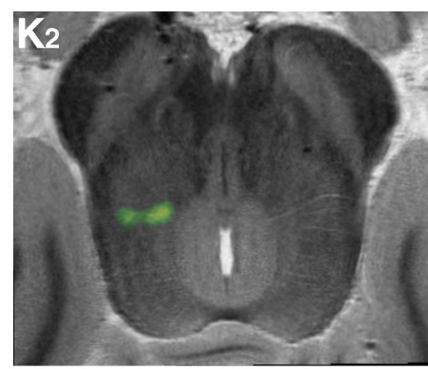

Tonic increase - PMJ +2 mm

Figure 5. Anatomical distribution of neuronal responses to locomotion. $\boldsymbol{A} \mathbf{1}-\mathbf{A} \mathbf{3}$ and $\mathbf{B 1}-\mathbf{B} \mathbf{3}$ display the localization of the phasic neurons of the two primates on $7 T$ MRI coronal and sagittal slices, respectively, perpendicular to the $\mathrm{AC}-\mathrm{PC}$ plane according to their stereotaxic coordinates. Phasic neurons are represented as red spheres and those responding to passive movement are represented as yellow spheres. Similarly, C1-C3 and D1-D3 display the localization of the tonic increase (green spheres), tonic decrease (violet spheres), and tonic prolonged increase (blue spheres) neurons of the two primates on 7T MRI in coronal and sagittal slices, respectively. Note that several neurons can be represented by one sphere. $\boldsymbol{E}-\mathbf{G}$, ChAT immunohistological localization of PPN cholinergic neurons within the MRF (Primate T) and equivalent 7T MRI sequences in coronal plane. Localization of the different images (MRI and ChAT) is expressed in relation to the anterior border of PC. E1, $\mathbf{F 1}$, and $\mathbf{G 1}$ are coronal MRI images acquired on $7 \mathrm{~T}$ MRI scanner from ex vivo brain. E3, F3, and $\mathbf{G 3}$ are enlargements of the dotted rectangles in $\mathbf{E 2}$, F2, and $\mathbf{G 2}$, respectively. Single arrowhead indicates the medial longitudinal fasciculus; double arrowheads, superior cerebellar peduncle; single white arrow, decussation of the superior peduncle; double white arrows, posterior commissure (posterior aspect); blue arrow, nucleus of the IV ${ }^{\text {th }}$ cranial nerve; red arrows, positive CHaT-labeled neurons. Aq, Aqueduct of Sylvius; $\mathbf{S C}$, superior colliculus. Scale bars, $1 \mathrm{~mm}$. $\boldsymbol{H}-\boldsymbol{K}$, Caudo-rostral distribution of the neuronal responses to locomotion in the transverse plane of the brainstem using map of density in the two primates. Transverse slices are expressed according to their distance from the PMJ. Orientation is provided in $\boldsymbol{H} \mathbf{1}$. Left images are always more caudal than the next image on the right. $\boldsymbol{H} \mathbf{1} \boldsymbol{K} \mathbf{1}$ and $\boldsymbol{H} \mathbf{2}-\boldsymbol{K} \mathbf{2}$ represent the density of phasic neurons (orange area) and tonic increase neurons (green area), respectively. $\mathbf{I}$ is an overlay representation on the two neuronal populations displayed in $\mathbf{I}$ and $\mathbf{I} \mathbf{2}$ at the level of the PMJ. Axis orientation is as follows: $P$, posterior; A, anterior; C, caudal; R, rostral.

lar-premamillary transection. Finally, it is likely that some responding neurons in the present study could also be involved in the control of posture by modulating muscle tone during locomotion (Mori, 1987; Lai and Siegel, 1990). Indeed, although the anatomical substrate of the control of muscle tone remains un- clear, different structures of the pontine and medullary reticular formation involved in the control of postural tone receive cholinergic and noncholinergic inputs from the MRF (Lai and Siegel, 1990, 1991; Lai et al., 1993; Takakusaki et al., 1996; Garcia-Rill et al., 2004). 
Table 3. Relative proportion of the responding neurons during locomotion (phasic and tonic) in the two primates according to their $X, Y$, and $Z$ coordinates in the different parts of the MRF

\begin{tabular}{|c|c|c|c|c|c|c|c|c|c|}
\hline & \multirow{3}{*}{ Unit $(n)$} & \multicolumn{8}{|l|}{ MRF } \\
\hline & & \multicolumn{4}{|l|}{ Medial } & \multicolumn{4}{|l|}{ Lateral } \\
\hline & & \multicolumn{2}{|l|}{ Anterior } & \multicolumn{2}{|c|}{ Posterior } & \multicolumn{2}{|c|}{ Anterior } & \multicolumn{2}{|c|}{ Posterior } \\
\hline Phasic & 58 & $27.6 \%$ & $29.6 \%$ & $6.9 \%$ & $6.9 \%$ & $1.7 \%$ & $19 \%$ & $1.7 \%$ & $6.9 \%$ \\
\hline Tonic & 122 & $17.2 \%$ & $11.5 \%$ & $23 \%$ & $13.1 \%$ & $4.9 \%$ & $9 \%$ & $11.5 \%$ & $9.8 \%$ \\
\hline
\end{tabular}

Nonresponder neurons are not included in the table.

Phasic neurons that responded to passive movement could be involved in sensorimotor processing due to the extensive dendrite arborization of the PPN neurons toward surrounding fiber tracts (Rye et al., 1987). Passive response of PPN neurons were also reported in decerebrated preparations and were suggested to receive spino-reticular input (Garcia-Rill and Skinner, 1988). Indeed, in primate, the spino-mesencephalic tractus and part of the spinoreticular tractus were found to reach different sites in the brainstem, including the caudal mesencephalon (Wiberg et al., 1987; Yezierski, 1988; Craig, 1995). Another origin of this passive response could be related to the close proximity with the SCP (Hazrati and Parent, 1992), which conveys information from the deep cerebellar nuclei, which is considered to be another locomotor center (Mori et al., 1999). The SCP also contains the ventral spino-cerebellar tractus thought to convey information about premotoneuronal activity in the cord. Indeed, recording during fictive locomotion in decerebrate cats showed that ventral spino-cerebellar tractus cells discharge in a phasic mode (Fedirchuk et al., 2013), similar to what we describe in the present study.

\section{Anatomo-functional description}

The different neurons that responded to locomotion were scattered throughout the caudal MRF at the level of the PMJ and never formed two segregated neuronal populations. This is consistent with studies showing that both PPN and CfN project to ponto-medullary reticular formation and spinal cord in primates (Castiglioni et al., 1978; Rolland et al., 2011). Although overlapping, a relative higher density of phasic neurons was found in the anterior part of the MRF, whereas the density of tonic neurons was higher in the posterior half of the MRF at the PMJ level. This latter region also showed a higher density of ChAT + neurons. In decerebrated preparations, a possible functional segregation in the MRF territories involved in locomotion was detected: rhythmic burst responses were found preferentially in the dorsal PPN, whereas the on/off cells were observed within the PPN pars compacta (Garcia-Rill and Skinner, 1988). Another studies in NHPs (Eidelberg et al., 1981) showed that the rostral part of the MRF (equivalent to dorsal in cat, including the CfN) was a site for inducing locomotion at low-threshold stimulation, whereas in humans, tonic responses to mimicked steps recorded during DBS implantation were found preferentially in the subcuneiform nucleus (Piallat et al., 2009).

\section{Interspecies considerations}

Althougth MER provides valuable functional data, caution should be exerted when interpreting FR analysis in primates (Israel and Bergman, 2008), which displays marked neuronal heterogeneity and a complex cytoarchitecture. The lack of a clear delineation between the PPN pars dissipata and the CfN in primates and humans is a further confounding factor. For this reason, our group and others use the terms "PPN area" or "MRF" to describe this region in clinical studies (Ferraye et al., 2010; Thevathasan et al., 2010; Alam et al., 2011) because most of the data are not restricted to the PPN per se, but rather also include the CfN. Therefore, comparisons between anatomical and functional data relative to the PPN in different species should be made with great caution (Edley and Graybiel, 1983; Alam et al., 2011). Furthermore, special attention should be paid to the variabilities in brainstem anatomy (brainstem orientation), the brainstem cutting plane, and the use of different anatomical planes to illustrate the data.

\section{Perspectives}

Regarding the involvement of primate MRF neurons during locomotion (present data), voluntary movement (Matsumura et al., 1997), and attentional process (Okada and Kobayashi, 2015), further electrophysiological studies at a different level of the motor command and the RAS will provide clues in the comprehension of motor control.

Interestingly, in primates, convergent projections from cortical areas (motor and premotor areas; Matsumura et al., 2000; Aravamuthan et al., 2007) and BG, particularly the different parts of the GPi and the SNr (Shink et al., 1997; Parent et al., 1999; Rolland et al., 2011), reach the MRF, suggesting that a single neuron potentially receives convergent inputs from the different functional BG loops (Shink et al., 1997) known to regulate goaldirected and automatic movements. Further studies will be needed to investigate whether MRF could be seen as a converging structure on the motor path, as has been hypothesized (Redgrave et al., 2010). This area of convergence on the motor path would also provide a modulatory control over the BG and brain states through strong excitatory projections to the SNc and intralaminar thalamic nuclei (Steriade et al., 1988; Lavoie and Parent, 1994b, 1994a).

Further studies investigating the role of the MRF neurons in locomotion, waking state, and transition from wakefulness to sleep in behaving primate are still needed. Indeed, an active role of the MRF neurons during locomotion and attentional process have not yet been described, but are expected in fine to provide new elements in the comprehension of the pathophysiology of gait disorders in PD known to be triggered by specific environmental context and cognitive state.

Finally, our study provides rational and anatomo-functional information for surgical strategies based on DBS electrode implantation in the MRF because a lack in the literature has been described regarding the functional role of the PPN/MRF in the locomotion and related disorders and its anatomical characteristic (Alam et al., 2011). It also demonstrates that single-unit activities can be recorded and extracted at the supraspinal level in behaving primate during locomotion, which might be of interest in the perspective of locomotor rehabilitation research (Noga et al., 2003; Bachmann et al., 2013). 


\section{References}

Abeles M (1982) Quantification, smoothing, and confidence limits for single-units' histograms. J Neurosci Methods 5:317-325. CrossRef Medline

Alam M, Schwabe K, Krauss JK (2011) The pedunculopontine nucleus area: critical evaluation of interspecies differences relevant for its use as a target for deep brain stimulation. Brain 134:11-23. CrossRef Medline

Aravamuthan BR, Muthusamy KA, Stein JF, Aziz TZ, Johansen-Berg H (2007) Topography of cortical and subcortical connections of the human pedunculopontine and subthalamic nuclei. Neuroimage 37:694-705. CrossRef Medline

Armstrong DM (1988) The supraspinal control of mammalian locomotion. J Physiol 405:1-37. CrossRef Medline

Bachmann LC, Matis A, Lindau NT, Felder P, Gullo M, Schwab ME (2013) Deep brain stimulation of the midbrain locomotor region improves paretic hindlimb function after spinal cord injury in rats. Sci Transl Med 5:208ra146-208ra146. Medline

Baron MS, Chaniary KD, Rice AC, Shapiro SM (2011) Multi-neuronal recordings in the basal ganglia in normal and dystonic rats. Front Syst Neurosci 5:67. Medline

Cant JG (1988) Positional behavior of long-tailed macaques (Macaca fascicularis) in northern Sumatra. Am J Phys Anthropol 76:29-37. CrossRef Medline

Castiglioni AJ, Gallaway MC, Coulter JD (1978) Spinal projections from the midbrain in monkey. J Comp Neurol 178:329-346. CrossRef Medline

Craig AD (1995) Distribution of brainstem projections from spinal lamina I neurons in the cat and the monkey. J Comp Neurol 361:225-248. CrossRef Medline

Devergnas A, Piallat B, Prabhu S, Torres N, Louis Benabid A, David O, Chabardès $S$ (2012) The subcortical hidden side of focal motor seizures: evidence from micro-recordings and local field potentials 135:2263-2276.

Drew T, Dubuc R, Rossignol S (1986) Discharge patterns of reticulospinal and other reticular neurons in chronic, unrestrained cats walking on a treadmill. J Neurophysiol 55:375-401. Medline

Edley SM, Graybiel AM (1983) The afferent and efferent connections of the feline nucleus tegmenti pedunculopontinus, pars compacta. J Comp Neurol 217:187-215. CrossRef Medline

Eidelberg E, Walden JG, Nguyen LH (1981a) Locomotor control in macaque monkeys. Brain 104:647-663. Medline

Favre J, Taha JM, Baumann T, Burchiel KJ (1999) Computer analysis of the tonic, phasic, and kinesthetic activity of pallidal discharges in Parkinson patients. Surg Neurol 51:665-672; discussion 672-673. Medline

Fedirchuk B, Stecina K, Kristensen KK, Zhang M, Meehan CF, Bennett DJ, Hultborn H (2013) Rhythmic activity of feline dorsal and ventral spinocerebellar tract neurons during fictive motor actions. J Neurophysiol 109: 375-388. CrossRef Medline

Fee MS, Mitra PP, Kleinfeld D (1996) Variability of extracellular spike waveforms of cortical neurons. J Neurophysiol 76:3823-3833. Medline

Ferraye MU, Debû B, Fraix V, Goetz L, Ardouin C, Yelnik J, Henry-Lagrange C, Seigneuret E, Piallat B, Krack P, Le Bas JF, Benabid AL, Chabardès S, Pollak P (2010) Effects of pedunculopontine nucleus area stimulation on gait disorders in Parkinson's disease. Brain 133:205-214. CrossRef Medline

Galvan A, Wichmann T (2008) Pathophysiology of parkinsonism. Clin Neurophysiol 119:1459-1474. CrossRef Medline

Garcia-Rill E (1991) The pedunculopontine nucleus. Prog Neurobiol 36: 363-389. CrossRef Medline

Garcia-Rill E (2015) Waking and the reticular activating system in health and disease. San Diego: Academic.

Garcia-Rill E, Skinner RD (1987a) The mesencephalic locomotor region. I. Activation of a medullary projection site. Brain Res 411:1-12. CrossRef Medline

Garcia-Rill E, Skinner RD (1987b) The mesencephalic locomotor region. II. Projections to reticulospinal neurons. Brain Res 411:13-20. CrossRef Medline

Garcia-Rill E, Skinner RD (1988) Modulation of rhythmic function in the posterior midbrain. Neuroscience 27:639-654. CrossRef Medline

Garcia-Rill E, Homma Y, Skinner RD (2004) Arousal mechanisms related to posture and locomotion: 1. Descending modulation. Prog Brain Res 143: 283-290. Medline

Goetz L, Piallat B, Thibaudier Y, Montigon O, David O, Chabardès S (2012) A nonhuman primate model of bipedal locomotion under restrained condition allowing gait studies and single unit brain recordings. J Neurosci Methods 204:306-317. CrossRef Medline

Grabli D, Karachi C, Welter ML, Lau B, Hirsch EC, Vidailhet M, François C (2012) Normal and pathological gait: what we learn from Parkinson's disease. J Neurol Neurosurg Psychiatry 83:979-985. CrossRef Medline

Hazrati LN, Parent A (1992) Projection from the deep cerebellar nuclei to the pedunculopontine nucleus in the squirrel monkey. Brain Res 585: 267-271. CrossRef Medline

Hemmi JM, Menzel C (1995) Foraging strategies of long-tailed macaques, Macaca fascicularis-directional extrapolation. Animal Behavior 49: 457-464. CrossRef

Inglis WL, Winn P (1995) The pedunculopontine tegmental nucleus: where the striatum meets the reticular formation. Prog Neurobiol 47:1-29. CrossRef Medline

Israel Z, Bergman H (2008) Pathophysiology of the basal ganglia and movement disorders: from animal models to human clinical applications. Neurosci Biobehav Rev 32:367-377. CrossRef Medline

Jordan LM (1998) Initiation of locomotion in mammals. Ann N Y Acad Sci 860:83-93. CrossRef Medline

Kang Y, Kitai ST (1990) Electrophysiological properties of pedunculopontine neurons and their postsynaptic responses following stimulation of substantia nigra reticulata. Brain Res 535:79-95. CrossRef Medline

Karachi C, Grabli D, Bernard FA, Tandé D, Wattiez N, Belaid H, Bardinet E, Prigent A, Nothacker HP, Hunot S, Hartmann A, Lehéricy S, Hirsch EC, François C (2010) Cholinergic mesencephalic neurons are involved in gait and postural disorders in Parkinson disease. J Clin Invest 120:27452754. CrossRef Medline

Kinomura S, Larsson J, Gulyás B, Roland PE (1996) Activation by attention of the human reticular formation and thalamic intralaminar nuclei. Science 271:512-515. CrossRef Medline

Kobayashi Y, Isa T (2002) Sensory-motor gating and cognitive control by the brainstem cholinergic system. Neural Netw 15:731-741. CrossRef Medline

Kobayashi Y, Inoue Y, Yamamoto M, Isa T, Aizawa H (2002) Contribution of pedunculopontine tegmental nucleus neurons to performance of visually guided saccade tasks in monkeys. J Neurophysiol 88:715-731. Medline

Kurland J (1973) A natural history of kra macaques (Macaca fascicularis Raffles, 1821) at the Kutai Reserve, Kalimantan Timur, Indonesia. Primates 14:245-262. CrossRef

Lai YY, Siegel JM (1990) Muscle tone suppression and stepping produced by stimulation of midbrain and rostral pontine reticular formation. J Neurosci 10:2727-2734. Medline

Lai YY, Siegel JM (1991) Pontomedullary glutamate receptors mediating locomotion and muscle tone suppression. J Neurosci 11:2931-2937. Medline

Lai YY, Clements JR, Siegel JM (1993) Glutamatergic and cholinergic projections to the pontine inhibitory area identified with horseradish peroxidase retrograde transport and immunohistochemistry. J Comp Neurol 336:321-330. CrossRef Medline

Lavoie B, Parent A (1994a) Pedunculopontine nucleus in the squirrel monkey: projections to the basal ganglia as revealed by anterograde tracttracing methods. J Comp Neurol 344:210-231. CrossRef Medline

Lavoie B, Parent A (1994b) Pedunculopontine nucleus in the squirrel monkey: cholinergic and glutamatergic projections to the substantia nigra. J Comp Neurol 344:232-241. CrossRef Medline

Lee AM, Hoy JL, Bonci A, Wilbrecht L, Stryker MP, Niell CM (2014) Identification of a brainstem circuit regulating visual cortical state in parallel with locomotion. Neuron 83:455-466. CrossRef Medline

Legéndy CR, Salcman M (1985) Bursts and recurrences of bursts in the spike trains of spontaneously active striate cortex neurons. J Neurophysiol 53: 926-939. Medline

Le Ray D, Juvin L, Ryczko D, Dubuc R (2011) Chapter 4-supraspinal control of locomotion: the mesencephalic locomotor region. Prog Brain Res 188:51-70. CrossRef Medline

Manaye KF, Zweig R, Wu D, Hersh LB, De Lacalle S, Saper CB, German DC (1999) Quantification of cholinergic and select non-cholinergic mesopontine neuronal populations in the human brain. Neuroscience 89: 759-770. CrossRef Medline

Martin RF, Bowden DM (1996) A stereotaxic template atlas of the macaque brain for digital imaging and quantitative neuroanatomy. Neuroimage 4:119-150. CrossRef Medline 
Martinez-Gonzalez C, Bolam JP, Mena-Segovia J (2011) Topographical organization of the pedunculopontine nucleus. Front Neuroanat 5:22. Medline

Martinez-Gonzalez C, Wang HL, Micklem BR, Bolam JP, Mena-Segovia J (2012) Subpopulations of cholinergic, GABAergic and glutamatergic neurons in the pedunculopontine nucleus contain calcium-binding proteins and are heterogeneously distributed. Eur J Neurosci 35:723-734. CrossRef Medline

Matsumura M, Watanabe K, Ohye C (1997) Single-unit activity in the primate nucleus tegmenti pedunculopontinus related to voluntary arm movement. Neurosci Res 28:155-165. CrossRef Medline

Matsumura M, Nambu A, Yamaji Y, Watanabe K, Imai H, Inase M, Tokuno $\mathrm{H}$, Takada M (2000) Organization of somatic motor inputs from the frontal lobe to the pedunculopontine tegmental nucleus in the macaque monkey. Neuroscience 98:97-110. CrossRef Medline

Mazzone P, Lozano A, Stanzione P, Galati S, Scarnati E, Peppe A, Stefani A (2005) Implantation of human pedunculopontine nucleus: a safe and clinically relevant target in Parkinson's disease. Neuroreport 16:18771881. CrossRef Medline

Mena-Segovia J, Winn P, Bolam JP (2008) Cholinergic modulation of midbrain dopaminergic systems. Brain Res Rev 58:265-271. CrossRef Medline

Mesulam MM, Mufson EJ, Levey AI, Wainer BH (1984) Atlas of cholinergic neurons in the forebrain and upper brainstem of the macaque based on monoclonal choline acetyltransferase immunohistochemistry and acetylcholinesterase histochemistry. Neuroscience 12:669-686. CrossRef Medline

Mesulam MM, Geula C, Bothwell MA, Hersh LB (1989) Human reticular formation: cholinergic neurons of the pedunculopontine and laterodorsal tegmental nuclei and some cytochemical comparisons to forebrain cholinergic neurons. J Comp Neurol 283:611-633. CrossRef Medline

Mori S (1987) Integration of posture and locomotion in acute decerebrate cats and in awake, freely moving cats. Prog Neurobiol 28:161-195. CrossRef Medline

Mori S, Matsui T, Kuze B, Asanome M, Nakajima K, Matsuyama K (1999) Stimulation of a restricted region in the midline cerebellar white matter evokes coordinated quadrupedal locomotion in the decerebrate cat. J Neurophysiol 82:290-300. Medline

Moruzzi G, Magoun HW (1949) Brain stem reticular formation and activation of the EEG. Electroencephalogr Clin Neurophysiol 1:455-473. CrossRef Medline

Noga BR, Kriellaars DJ, Brownstone RM, Jordan LM (2003) Mechanism for activation of locomotor centers in the spinal cord by stimulation of the mesencephalic locomotor region. J Neurophysiol 90:1464-1478. CrossRef Medline

Okada K, Kobayashi Y (2015) Rhythmic firing of pedunculopontine tegmental nucleus neurons in monkeys during eye movement task. PLoS One 10:e0128147. CrossRef Medline

Pahapill PA, Lozano AM (2000) The pedunculopontine nucleus and Parkinson's disease. Brain 123:1767-1783. CrossRef Medline

Parent M, Lévesque M, Parent A (1999) The pallidofugal projection system in primates: evidence for neurons branching ipsilaterally and contralaterally to the thalamus and brainstem. J Chem Neuroanat 16:153-165. CrossRef Medline

Percheron G (1997) A reliable system of ventricular coordinates for the cartography and stereotaxy of the amygdala (and anterior hippocampus) in macaques. J Neurosci Methods 75:5-14. CrossRef Medline

Pereira EA, Nandi D, Jenkinson N, Stein JF, Green AL, Aziz TZ (2011) Pedunculopontine stimulation from primate to patient. J Neural Transm 118:1453-1460. CrossRef Medline

Piallat B, Chabardès S, Torres N, Fraix V, Goetz L, Seigneuret E, Bardinet E, Ferraye M, Debu B, Krack P, Yelnik J, Pollak P, Benabid AL (2009) Gait is associated with an increase in tonic firing of the sub-cuneiform nucleus neurons. Neuroscience 158:1201-1205. CrossRef Medline

Piallat B, Polosan M, Fraix V, Goetz L, David O, Fenoy A, Torres N, Quesada JL, Seigneuret E, Pollak P, Krack P, Bougerol T, Benabid AL, Chabardès S (2011) Subthalamic neuronal firing in obsessive-compulsive disorder and Parkinson disease. Ann Neurol 69:793-802. CrossRef Medline

Plaha P, Gill SS (2005) Bilateral deep brain stimulation of the pedunculopontine nucleus for Parkinson's disease. Neuroreport 16:1883-1887. CrossRef Medline
Redgrave P, Rodriguez M, Smith Y, Rodriguez-Oroz MC, Lehéricy S, Bergman H, Agid Y, DeLong MR, Obeso JA (2010) Goal-directed and habitual control in the basal ganglia: implications for Parkinson's disease. Nat Rev Neurosci 11:760-772. CrossRef Medline

Rolland AS, Tandé D, Herrero MT, Luquin MR, Vazquez-Claverie M, Karachi C, Hirsch EC, François C (2009) Evidence for a dopaminergic innervation of the pedunculopontine nucleus in monkeys, and its drastic reduction after MPTP intoxication. J Neurochem 110:1321-1329. CrossRef Medline

Rolland AS, Karachi C, Muriel MP, Hirsch EC, François C (2011) Internal pallidum and substantia nigra control different parts of the mesopontine reticular formation in primate. Mov Disord 26:1648-1656. CrossRef Medline

Ros H, Magill PJ, Moss J, Bolam JP, Mena-Segovia J (2010) Distinct types of non-cholinergic pedunculopontine neurons are differentially modulated during global brain states. Neuroscience 170:78-91. CrossRef Medline

Ryczko D, Dubuc R (2013) The multifunctional mesencephalic locomotor region. Curr Pharm Des 19:4448-4470. CrossRef Medline

Rye DB, Saper CB, Lee HJ, Wainer BH (1987) Pedunculopontine tegmental nucleus of the rat: cytoarchitecture, cytochemistry, and some extrapyramidal connections of the mesopontine tegmentum. J Comp Neurol 259: 483-528. CrossRef Medline

Shik ML, Severin FV, Orlovskiü GN (1966) [Control of walking and running by means of electric stimulation of the midbrain]. Biofizika 11:659-666. Medline

Shimamoto SA, Larson PS, Ostrem JL, Glass GA, Turner RS, Starr PA (2010) Physiological identification of the human pedunculopontine nucleus. J Neurol Neurosurg Psychiatr 81:80-86. CrossRef Medline

Shink E, Sidibé M, Smith Y (1997) Efferent connections of the internal globus pallidus in the squirrel monkey: II. Topography and synaptic organization of pallidal efferents to the pedunculopontine nucleus. J Comp Neurol 382:348-363. Medline

Sinnamon HM (1993) Preoptic and hypothalamic neurons and the initiation of locomotion in the anesthetized rat. Prog Neurobiol 41:323-344. CrossRef Medline

Steriade M (1996) Awakening the brain. Nature 383:24-25. CrossRef Medline

Steriade M, Paré D, Parent A, Smith Y (1988) Projections of cholinergic and non-cholinergic neurons of the brainstem core to relay and associational thalamic nuclei in the cat and macaque monkey. Neuroscience 25:47-67. CrossRef Medline

Takakusaki K, Kitai ST (1997) Ionic mechanisms involved in the spontaneous firing of tegmental pedunculopontine nucleus neurons of the rat. Neuroscience 78:771-794. CrossRef Medline

Takakusaki K, Shiroyama T, Yamamoto T, Kitai ST (1996) Cholinergic and noncholinergic tegmental pedunculopontine projection neurons in rats revealed by intracellular labeling. J Comp Neurol 371:345-361. CrossRef Medline

Thevathasan W, Silburn PA, Brooker H, Coyne TJ, Khan S, Gill SS, Aziz TZ, Brown P (2010) The impact of low-frequency stimulation of the pedunculopontine nucleus region on reaction time in parkinsonism. J Neurol Neurosurg Psychiatr 81:1099-1104. CrossRef Medline

Wang HL, Morales M (2009) Pedunculopontine and laterodorsal tegmental nuclei contain distinct populations of cholinergic, glutamatergic and GABAergic neurons in the rat. Eur J Neurosci 29:340-358. CrossRef Medline

Weinberger M, Hamani C, Hutchison WD, Moro E, Lozano AM, Dostrovsky JO (2008) Pedunculopontine nucleus microelectrode recordings in movement disorder patients. Exp Brain Res 188:165-174. CrossRef Medline

Wiberg M, Westman J, Blomqvist A (1987) Somatosensory projection to the mesencephalon: an anatomical study in the monkey. J Comp Neurol 264:92-117. CrossRef Medline

Wichmann T, Bergman H, DeLong MR (1994) The primate subthalamic nucleus. I. Functional properties in intact animals. J Neurophysiol 72 : 494-506. Medline

Yezierski RP (1988) Spinomesencephalic tract: projections from the lumbosacral spinal cord of the rat, cat, and monkey. J Comp Neurol 267:131146. CrossRef Medline 